\title{
On the closure of the Hodge locus of positive period dimension
}

\author{
B. Klingler ${ }^{1}$ - A. Otwinowska ${ }^{1}$
}

Received: 30 March 2019 / Accepted: 4 March 2021 / Published online: 29 March 2021

(C) The Author(s) 2021

\begin{abstract}
Given $\mathbb{V}$ a polarizable variation of $\mathbb{Z}$-Hodge structures on a smooth connected complex quasi-projective variety $S$, the Hodge locus for $\mathbb{V}^{\otimes}$ is the set of closed points $s$ of $S$ where the fiber $\mathbb{V}_{s}$ has more Hodge tensors than the very general one. A classical result of Cattani, Deligne and Kaplan states that the Hodge locus for $\mathbb{V}^{\otimes}$ is a countable union of closed irreducible algebraic subvarieties of $S$, called the special subvarieties of $S$ for $\mathbb{V}$. Under the assumption that the adjoint group of the generic Mumford-Tate group of $\mathbb{V}$ is simple we prove that the union of the special subvarieties for $\mathbb{V}$ whose image under the period map is not a point is either a closed algebraic subvariety of $S$ or is Zariski-dense in $S$. This implies for instance the following typical intersection statement: given a Hodge-generic closed irreducible algebraic subvariety $S$ of the moduli space $\mathcal{A}_{g}$ of principally polarized Abelian varieties of dimension $g$, the union of the positive dimensional irreducible components of the intersection of $S$ with the strict special subvarieties of $\mathcal{A}_{g}$ is either a closed algebraic subvariety of $S$ or is Zariski-dense in $S$.
\end{abstract}

B.K.'s research is supported by an Einstein Foundation's professorship.

$\triangle$ B. Klingler

bruno.klingler@hu-berlin.de

A. Otwinowska

ania.otwinowska@hu-berlin.de

1 Humboldt Universität zu Berlin, Berlin, Germany 


\section{Introduction}

\subsection{Motivation: Hodge loci}

Let $\left(\mathbb{V}_{\mathbb{Z}}, \mathcal{V}, F^{\bullet}, \nabla\right)$ be a polarizable variation of $\mathbb{Z}$-Hodge structure ( $\mathbb{Z} \mathrm{VHS}$ ) of arbitrary weight on a smooth connected complex quasi-projective variety $S$. Thus $\mathbb{V}_{\mathbb{Z}}$ is a finite rank locally free $\mathbb{Z}_{S \text { an }}$-local system on the complex manifold $S^{\text {an }}$ associated to $S$; and $\left(\mathcal{V}, F^{\bullet}, \nabla\right)$ is the unique algebraic regular filtered flat connection on $S$ whose analytification is $\mathbb{V} \otimes_{\mathbb{Z}_{S} \text { an }} \mathcal{O}_{S}$ an endowed with its Hodge filtration $F^{\bullet}$ and the holomorphic flat connection $\nabla^{\text {an }}$ defined by $\mathbb{V}$, see [23, (4.13)]). From now on we will abbreviate the $\mathbb{Z} V H S\left(\mathbb{V}_{\mathbb{Z}}, \mathcal{V}, F^{\bullet}, \nabla\right)$ simply by $\mathbb{V}$. A typical example is the weight zero polarizable $\mathbb{Z V H S}$ "of geometric origin"

$$
\left(\mathbb{V}_{\mathbb{Z}}:=R^{2 k} f_{*}^{\text {an }} \mathbb{Z}(k) /(\text { torsion }), \mathcal{V}:=R^{2 k} f_{*} \Omega_{X / S}^{\bullet}, F^{\bullet}, \nabla\right)
$$

associated to a smooth projective morphism of smooth irreducible complex quasi-projective varieties $f: X \rightarrow S$. In this case the Hodge filtration $F^{\bullet}$ is induced by the stupid filtration on the algebraic De Rham complex $\Omega_{X / S}^{\bullet}$ and $\nabla$ is the Gauß-Manin connection.

The Hodge locus $\operatorname{HL}(S, \mathbb{V})$ is the set of points $s \in S^{\text {an }}$ for which the Hodge structure $\mathbb{V}_{S}$ admits more Hodge classes than the very general fiber $\mathbb{V}_{s^{\prime}}$ (for us a Hodge class of a pure $\mathbb{Z}$-Hodge structure $H=\left(H_{\mathbb{Z}}, F^{\bullet}\right.$ ) is a class in $H_{\mathbb{Z}}$ whose image in $H_{\mathbb{C}}$ lies in $F^{0} H_{\mathbb{C}}$, or equivalently a morphism of Hodge structures $\mathbb{Z}(0) \rightarrow H$ ). It is empty if $\mathbb{V}$ contains no non-trivial weight zero factor. More generally let $\mathbb{V}^{\otimes}$ be the countable direct sum of polarizable $\mathbb{Z}$ VHSs $\bigoplus_{a, b \in \mathbb{N}} \mathbb{V}^{\otimes a} \otimes\left(\mathbb{V}^{\vee}\right)^{\otimes b}$ (where $\mathbb{V}^{\vee}$ denotes the $\mathbb{Z}$ VHS dual of $\mathbb{V}$ ). The Hodge locus $\operatorname{HL}\left(S, \mathbb{V}^{\otimes}\right)$ is the subset of points $s \in S^{\text {an }}$ for which the Hodge structure $\mathbb{V}_{s}$ admits more Hodge tensors than the very general fiber $\mathbb{V}_{s^{\prime}}$. It contains $\operatorname{HL}(S, \mathbb{V})$, usually strictly.

In the geometric case Weil [28] asked whether $\operatorname{HL}(S, \mathbb{V})$ is a countable union of closed algebraic subvarieties of $S$ (he noticed that a positive answer follows easily from the rational Hodge conjecture). In [4] Cattani, Deligne and Kaplan proved the following unconditional celebrated result (see [5], we also refer to [3] for an alternative proof):

Theorem 1.1 (Cattani-Deligne-Kaplan) Let $S$ be a smooth connected complex quasi-projective algebraic variety and $\mathbb{V}$ be a polarizable $\mathbb{Z} V H S$ over $S$. Then $\operatorname{HL}(S, \mathbb{V})$ (thus also $\operatorname{HL}\left(S, \mathbb{V}^{\otimes}\right)$ ) is a countable union of closed irreducible algebraic subvarieties of $S$.

The locus $\operatorname{HL}\left(S, \mathbb{V}^{\otimes}\right)$ is easier to understand than $\operatorname{HL}(S, \mathbb{V})$ as it has a grouptheoretical interpretation. Recall that the Mumford-Tate group MT $(H) \subset$ 
$\mathbf{G L}(H)$ of a $\mathbb{Q}$-Hodge structure $H$ is the Tannakian group of the Tannakian category $\left\langle H^{\otimes}\right\rangle$ of $\mathbb{Q}$-Hodge structures tensorially generated by $H$ and its dual $H^{\vee}$. Equivalently, the group $\mathbf{M T}(H)$ is the fixator in $\mathbf{G L}(H)$ of the Hodge tensors for $H$. Given a polarized $\mathbb{Z}$ VHS $\mathbb{V}$ on $S$ as above and $Y \hookrightarrow S$ a closed irreducible algebraic subvariety, a point $s$ of $Y^{\text {an }}$ is said to be Hodge-generic in $Y$ for $\mathbb{V}$ if $\mathbf{M T}\left(\mathbb{V}_{s, \mathbb{Q}}\right)$ has maximal dimension when $s$ ranges through $Y^{\text {an }}$. Two Hodge-generic points in $Y^{\text {an }}$ for $\mathbb{V}$ have the same Mumford-Tate group, called the generic Mumford-Tate group $\mathbf{M T}\left(Y, \mathbb{V}_{\mid Y}\right)$ of $Y$ for $\mathbb{V}$. The Hodge locus $\operatorname{HL}\left(S, \mathbb{V}^{\otimes}\right)$ is also the subset of points of $S$ which are not Hodge-generic in $S$ for $\mathbb{V}$.

Definition 1.2 A special subvariety of $S$ for $\mathbb{V}$ is a closed irreducible algebraic subvariety $Y \subset S$ maximal among the closed irreducible algebraic subvarieties $Z$ of $S$ such that $\mathbf{M T}\left(Z, \mathbb{V}_{\mid Z}\right)=\mathbf{M T}\left(Y, \mathbb{V}_{\mid Y}\right)$.

In particular $S$ is always special for $\mathbb{V}$. Theorem 1.1 for $\operatorname{HL}\left(S, \mathbb{V}^{\otimes}\right)$ can be rephrased by saying that the set of special subvarieties of $S$ for $\mathbb{V}$ is countable and that $\operatorname{HL}\left(S, \mathbb{V}^{\otimes}\right)$ is the (countable) union of the strict special subvarieties of $S$ for $\mathbb{V}$.

\subsection{Main result}

In this paper we investigate the geometry of the Zariski-closure of the Hodge locus $\operatorname{HL}\left(S, \mathbb{V}^{\otimes}\right)$. Our methods are variational, hence we only detect the special subvarieties of $S$ for $\mathbb{V}$ which are of positive period dimension in the following sense:

Definition 1.3 A closed irreducible subvariety $Y$ of $S$ is said to be of positive period dimension for $\mathbb{V}$ if the local system $\mathbb{V}_{\mid Y}$ is not isotrivial.

Equivalently, $Y$ is of positive period dimension for $\mathbb{V}$ if and only if its algebraic monodromy group $\mathbf{H}_{Y}$ for $\mathbb{V}$ (see Definition 2.1) is not equal to $\{1\}$; or equivalently if the period map $\Phi_{S}: S^{\text {an }} \rightarrow \Gamma \backslash \mathcal{D}^{+}$describing $\mathbb{V}^{\otimes}$ (see Sect. 4) does not contract $Y^{\text {an }}$ to a point in the connected Hodge variety $\Gamma \backslash \mathcal{D}^{+}$. When $\mathbb{V}$ satisfies the infinitesimal Torelli condition (i.e. the period map $\Phi_{S}$ is an immersion), a closed irreducible subvariety $Y$ of $S$ is of positive period dimension for $\mathbb{V}$ if and only if it is positive dimensional.

Definition 1.4 We define the Hodge locus of positive period dimension $\operatorname{HL}\left(S, \mathbb{V}^{\otimes}\right)_{\text {pos }} \subset \operatorname{HL}\left(S, \mathbb{V}^{\otimes}\right)$ as the union of the strict special subvarieties of $S$ for $\mathbb{V}$ which are of positive period dimension for $\mathbb{V}$.

Our main result describes the Zariski-closure of $\operatorname{HL}\left(S, \mathbb{V}^{\otimes}\right)_{\text {pos }}$ : 
Theorem 1.5 Let $\mathbb{V}$ be a polarizable $\mathbb{Z} V H S$ on a smooth connected complex quasi-projective variety $S$. Suppose that the adjoint group of the generic Mumford-Tate group $\mathbf{M T}(S, \mathbb{V})$ is simple (we will say that $\mathbf{M T}(S, \mathbb{V})$ is non-product). Then either $\operatorname{HL}\left(S, \mathbb{V}^{\otimes}\right)_{\text {pos }}$ is a finite union of strict special subvarieties of $S$; or it is Zariski-dense in $S$.

In other words: either the set of strict special subvarieties of $S$ for $\mathbb{V}$ which are of positive period dimension for $\mathbb{V}$ has finitely many maximal elements (for the inclusion); or the union of such special subvarieties is Zariski-dense in $S$.

\subsection{Examples}

Theorem 1.5 is new even in the much-studied case where the $\mathbb{Z V H S} \mathbb{V}$ has weight 1 or 2 . Let us warn the reader that these cases, which are simpler to describe, are not representative: in higher weight we expect $\operatorname{HL}\left(S, \mathbb{V}^{\otimes}\right)_{\text {pos }}$ to be algebraic in general.

\subsubsection{Example 1: subvarieties of Shimura varieties}

Let $\operatorname{Sh}_{K}^{0}(\mathbf{G}, X)$ be a connected Shimura variety associated to a Shimura datum $(\mathbf{G}, X)$, with $\mathbf{G}$ non-product, and a level $K$ chosen to be neat (we refer to [18] for a nice survey on Shimura varieties). For $(\mathbf{G}, X)=\left(\mathbf{G S p}(2 g), \mathbf{H}_{g}\right)$, $g \geq 1$, the Shimura variety $\operatorname{Sh}_{K}^{0}(\mathbf{G}, X)$ is the moduli space $\mathcal{A}_{g}$ of principally polarized Abelian varieties of dimension $g$ (endowed with some additional level structure). Let $\mathbb{V}$ be the $\mathbb{Z}$ VHS on $\operatorname{Sh}_{K}^{0}(\mathbf{G}, X)$ associated to a faithful rational representation of $\mathbf{G}$ (see $[12,3.2]$ ). The Hodge locus $\operatorname{HL}\left(\operatorname{Sh}_{K}^{0}(\mathbf{G}, X)\right):=\operatorname{HL}\left(\operatorname{Sh}_{K}^{0}(\mathbf{G}, X), \mathbb{V}^{\otimes}\right)$ is well-known to be independent of the choice of the faithful representation and is completely described in terms of Shimura subdata of $(\mathbf{G}, X)$, see [19]. The special points of $\operatorname{Sh}_{K}^{0}(\mathbf{G}, X)$, i.e. the special subvarieties of dimension zero, are the CM-points, i.e. the points of $\operatorname{Sh}_{K}^{0}(\mathbf{G}, X)$ whose Mumford-Tate group is commutative. In the case of $\mathcal{A}_{g}$ the CM-points correspond to abelian varieties with complex multiplication. Any connected Shimura variety contains an analytically dense set of special points (see [18, Lemma 3.3 and 3.5]), in particular $\operatorname{HL}\left(\operatorname{Sh}_{K}^{0}(\mathbf{G}, X)\right.$ ) is analytically dense in $\operatorname{Sh}_{K}^{0}(\mathbf{G}, X)$. The same proof shows that $\operatorname{HL}\left(\operatorname{Sh}_{K}^{0}(\mathbf{G}, X)\right)_{\text {pos }}$ is analytically dense in $\operatorname{Sh}_{K}^{0}(\mathbf{G}, X)$ as soon as it is not empty. For instance $\mathrm{HL}\left(\mathcal{A}_{g}\right)_{\text {pos }}$ is analytically dense in $\mathcal{A}_{g}$.

Remark 1.6 There exist Shimura varieties whose positive dimensional Hodge locus is empty, for instance the Kottwitz unitary Shimura varieties (see [8]) obtained by taking for $\mathbf{G}$ the group of invertible elements of a division algebra 
of prime degree endowed with an involution of the second kind. Ball quotients of Kottwitz type are the simplest examples.

If $S \subset \operatorname{Sh}_{K}^{0}(\mathbf{G}, X)$ is a closed irreducible subvariety the special subvarieties of $S$ for $\mathbb{V}_{\mid S}$ are precisely the irreducible components of the intersection of $S$ with the special subvarieties of $\operatorname{Sh}_{K}^{0}(\mathbf{G}, X)$. Theorem 1.5 thus implies immediately:

Corollary 1.7 Let $\operatorname{Sh}_{K}^{0}(\mathbf{G}, X)$ be a smooth connected Shimura variety associated to a Shimura datum $(\mathbf{G}, X)$ with $\mathbf{G}$ non-product. Let $S \subset \operatorname{Sh}_{K}^{0}(\mathbf{G}, X)$ be a closed irreducible subvariety which is Hodge generic (i.e. $\left.\mathbf{M T}\left(S, \mathbb{V}_{\mid S}\right)=\mathbf{G}\right)$ ). Either the positive dimensional irreducible components of the intersection of $S$ with the strict special subvarieties of $\operatorname{Sh}_{K}^{0}(\mathbf{G}, X)$ form a set with finitely many maximal elements (for the inclusion), or their union is Zariski-dense in $S$.

In the case of $\operatorname{Sh}_{K}^{0}(\mathbf{G}, X)=\mathcal{A}_{g}$ this reads:

Corollary 1.8 Let $S \subset \mathcal{A}_{g}$ be a Hodge-generic closed irreducible subvariety. Either the set of positive dimensional closed irreducible subvarieties of $S$ which are not Hodge generic has finitely many maximal elements (for the inclusion), or their union is Zariski-dense in S.

Corollary 1.7, which describes the distribution of all positive dimensional intersections of the Hodge generic $S$ with the special subvarieties of $\mathrm{Sh}_{K}^{0}(\mathbf{G}, X)$, should be compared with the classical André-Oort conjecture, which predicts under the same hypotheses that there are only finitely many special subvarieties of $\operatorname{Sh}_{K}^{0}(\mathbf{G}, X)$ contained in $S$ and maximal for these properties. The André-Oort conjecture has been proven when $\operatorname{Sh}_{K}^{0}(\mathbf{G}, X)$ is of abelian type, for instance for $\operatorname{Sh}_{K}^{0}(\mathbf{G}, X)=\mathcal{A}_{g}$. We refer to [17] for a survey on the André-Oort conjecture. While the André-Oort conjecture is an "atypical intersection" statement in the sense of [29], Corollary 1.7 may be thought of as a "typical intersection" statement. In particular both statements seem completely independent.

More generally Theorem 1.5 is the "typical intersection" counterpart to the "atypical intersection" conjecture for $\mathbb{Z}$ VHS proposed in [15, Conj. 1.9] (which generalizes the Zilber-Pink conjectures for Shimura varieties). It provides an answer to the geometric part of the naïve [15, Question 1.2] (we warn the reader that our $\operatorname{HL}\left(S, \mathbb{V}^{\otimes}\right)$ is denoted $\operatorname{HL}(S, \mathbb{V})$ in [15]).

Even in the setting of Corollary 1.7 or Corollary 1.8, we don't know of any simple criterion for deciding whether $\operatorname{HL}\left(S, \mathbb{V}_{\mid S}^{\otimes}\right)_{\text {pos }}$ is a strict closed algebraic subvariety of $S$ or Zariski-dense in $S$. For $\operatorname{Sh}_{K}^{0}(\mathbf{G}, X)=\mathcal{A}_{g}$, Izadi [14], following ideas of [9], proved that $\operatorname{HL}\left(S, \mathbb{V}_{\mid S}^{\otimes}\right)$ is analytically (hence Zariski-) dense in $S$ for any irreducible $S \subset \mathcal{A}_{g}$ of codimension at most $g$. Her 
proof adapts immediately to show that $\operatorname{HL}\left(S, \mathbb{V}_{\mid S}^{\otimes}\right)_{\text {pos }}$ is analytically dense in $S$ if $S$ has codimension at most $g-1$. Generalizing the results of [14] to a general connected Shimura variety $\operatorname{Sh}_{K}^{0}(\mathbf{G}, X)$, Chai (see [6]) showed the following. Let $\mathbf{H} \subset \mathbf{G}$ be a Hodge subgroup. Let $\operatorname{HL}\left(S, \mathbb{V}^{\otimes}, \mathbf{H}\right) \subset \operatorname{HL}\left(S, \mathbb{V}^{\otimes}\right)$ denote the subset of points $s \in S$ whose Mumford-Tate group $\mathbf{M T}_{s}(\mathbb{V})$ is $\mathbf{G}(\mathbb{Q})$ conjugated to $\mathbf{H}$. Then there exists an explicit constant $c(\mathbf{G}, X, \mathbf{H}) \in \mathbb{N}$, whose value is $g$ in the example above, which has the property that $\operatorname{HL}\left(S, \mathbb{V}^{\otimes}, \mathbf{H}\right)$, hence also $\operatorname{HL}\left(S, \mathbb{V}^{\otimes}\right)$ is analytically dense in $S$ as soon as $S$ has codimension at most $c(\mathbf{G}, X, \mathbf{H})$ in $\operatorname{Sh}_{K}(\mathbf{G}, X)$. Once more it follows from the analysis of the proof of [6] that $\operatorname{HL}\left(S, \mathbb{V}^{\otimes}\right)_{\text {pos }}$ is analytically dense in $S$ as soon as $S$ has codimension at most $c(\mathbf{G}, X, \mathbf{H})-1$.

\subsubsection{Example 2: classical Noether-Lefschetz locus}

Let $B \subset \mathbb{P} H^{0}\left(\mathbb{P}_{\mathbb{C}}^{3}, \mathcal{O}(d)\right)$ be the open subvariety parametrizing the smooth surfaces of degree $d$ in $\mathbb{P}_{\mathbb{C}}^{3}$. From now on we suppose $d>3$. The classical Noether theorem states that any surface $Y \subset \mathbb{P}_{\mathbb{C}}^{3}$ corresponding to a very general point $[Y] \in B$ has Picard group $\mathbb{Z}$ : every curve on $Y$ is a complete intersection of $Y$ with another surface in $\mathbb{P}_{\mathbb{C}}^{3}$. The countable union $\operatorname{NL}(B)$ of closed algebraic subvarieties of $B$ corresponding to surfaces with bigger Picard group is called the Noether-Lefchetz locus of $B$. Let $\mathbb{V} \rightarrow B$ be the $\mathbb{Z}$ VHS $R^{2} f_{*} \mathbb{Z}$, where $f: \mathcal{Y} \rightarrow B$ denotes the universal family of surfaces of degree $d$. Clearly $\operatorname{NL}(B) \subset \operatorname{HL}\left(B, \mathbb{V}^{\otimes}\right)$. Green (see [26, Prop.5.20]) proved that $\mathrm{NL}(B)$ is analytically dense in $B$ (see also [7] for a weaker result). In particular $\operatorname{HL}\left(B, \mathbb{V}^{\otimes}\right)$ is dense in $B$. Once more the analysis of Green's proof shows that in fact $\operatorname{HL}\left(B, \mathbb{V}^{\otimes}\right)_{\text {pos }}$ is dense in $B$. Now Theorem 1.5 implies the following:

Corollary 1.9 Let $S \subset B$ be a Hodge-generic closed irreducible subvariety. Either $S \cap \mathrm{HL}\left(B, \mathbb{V}^{\otimes}\right)_{\text {pos }}$ contains only finitely many maximal positive dimensional closed irreducible subvarieties of $S$, or the union of such subvarieties is Zariski-dense in $\mathrm{S}$.

Remark 1.10 We don't know if Corollary 1.9 remains true if we replace $\operatorname{HL}\left(B, \mathbb{V}^{\otimes}\right)_{\text {pos }}$ with $\mathrm{NL}(B)$.

\subsection{Organization of the paper}

The next Sect. 2 introduce the basic notation concerning local systems and $\mathbb{Z V H S}$ we will need. Section 3 then describes the main ingredients and the general strategy for proving Theorem 1.5. The reader will find at the end of Sect. 3 the organization of the rest of the paper. 


\section{Some notation}

\subsection{Notation for local systems}

Let $S$ be a smooth connected complex quasi-projective variety. Let $\mathbb{V}_{\mathbb{Z}}$ be a finite rank locally free $\mathbb{Z}$-local system on $S$ and $(\mathcal{V}, \nabla)$ the regular algebraic connection on $S$ [11, Theor. 5.9] associated to $\mathbb{V}_{\mathbb{Z}}$.

The local system $\mathbb{V}_{\mathbb{Z}}$ can be uniquely written as $\tilde{S} \times_{\rho} V_{\mathbb{Z}}$, where $\pi: \tilde{S} \rightarrow S$ denotes the complex analytic universal cover of $S$ associated to the choice of a point $s_{0}$ in $S, V_{\mathbb{Z}}:=H^{0}\left(\tilde{S}, \pi^{-1} \mathbb{V}_{\mathbb{Z}}\right) \simeq \mathbb{V}_{s_{0}, \mathbb{Z}}$ is a free $\mathbb{Z}$-module of finite rank and $\rho: \pi_{1}\left(S, s_{0}\right) \rightarrow \mathbf{G L}\left(V_{\mathbb{Z}}\right)$ denotes the monodromy representation of the local system $\mathbb{V}_{\mathbb{Z}}$. This corresponds to a complex analytic trivialization of $\tilde{\mathcal{V}}:=\mathcal{V} \times{ }_{S} \tilde{S}$ as a product $\tilde{S} \times V$, where $V:=V_{\mathbb{Z}} \otimes_{\mathbb{Z}} \mathbb{C}$. We still let $\pi: \tilde{S} \times V \rightarrow \mathcal{V}$ denote the natural projection. Recall the following classical definition:

Definition 2.1 Given a closed irreducible algebraic subvariety $i: Y \hookrightarrow S$, let $n: Y^{\text {nor }} \rightarrow Y$ be its normalisation. The algebraic monodromy group $\mathbf{H}_{Y}$ of $Y$ for $\mathbb{V}_{\mathbb{Z}}$ is the (conjugacy class of the) identity component of the Zariski-closure in $\mathbf{G L}\left(V_{\mathbb{Q}}\right)$ of the monodromy of the restriction to $Y^{\text {nor }}$ of the local system $n^{*} \mathbb{V}_{\mathbb{Z}}$.

Definition 2.2 Given $\lambda=\pi\left(\tilde{s}, \lambda_{0}\right) \in \mathcal{V}$ we define $\mathbb{V}(\lambda):=\pi\left(\tilde{S} \times\left\{\lambda_{0}\right\}\right) \subset \mathcal{V}$ the flat leaf of $\lambda$ for $\nabla$.

The set $\mathbb{V}(\lambda)$ is naturally a connected closed complex analytic subspace of the étalé space of the complex local system $\mathbb{V}_{\mathbb{C}}:=\mathbb{V}_{\mathbb{Z}} \otimes_{\mathbb{Z}} \mathbb{C}$. We will always endow $\mathbb{V}(\lambda)$ with its reduced analytic structure. When $\lambda=\pi\left(\tilde{s}, \lambda_{0}\right)$ is not a complex multiple of an element of $\mathbb{V}_{\mathbb{Z}}$, the orbit of $\lambda_{0}$ in $V$ under the monodromy group $\rho\left(\pi_{1}\left(S, s_{0}\right)\right) \subset \mathbf{G L}(V)$ has usually accumulation points, in which case $\mathbb{V}(\lambda)$ is not an analytic subvariety of $\mathcal{V}$.

\subsection{Notation for $\mathbb{Z V H S}$}

Suppose now that $\mathbb{V}:=\left(\mathbb{V}_{\mathbb{Z}}, \mathcal{V}, F^{\bullet}, \nabla\right)$ is a $\mathbb{Z}$ VHS on $S$. All $\mathbb{Z}$ VHS are assumed to be polarizable. In particular the algebraic monodromy group $\mathbf{H}_{S}$ is semi-simple.

Definition 2.3 Let $\lambda \in \mathcal{V}$ and $i \in \mathbb{Z}$. The locus of classes of $F^{i}$-type $\mathbb{V}^{i}(\lambda)$ for $\lambda$ is the intersection of the flat leaf $\mathbb{V}(\lambda)$ with $F^{i} \mathcal{V}$ :

$$
\mathbb{V}^{i}(\lambda):=\mathbb{V}(\lambda) \cap F^{i} \mathcal{V} \subset F^{i} \mathcal{V}
$$


The locus of $F^{i}$-type for $\lambda$ is the projection

$$
S^{i}(\lambda):=p\left(\mathbb{V}^{i}(\lambda)\right) \subset S .
$$

Again, $\mathbb{V}^{i}(\lambda)$ is naturally a complex analytic subspace (possibly with infinitely many connected components) of the étalé space of the complex local system $\mathbb{V}_{\mathbb{C}}:=\mathbb{V}_{\mathbb{Z}} \otimes_{\mathbb{Z}} \mathbb{C}$. When $\lambda$ is not a complex multiple of an element of $\mathbb{V}_{\mathbb{C}}$ the complex space $\mathbb{V}^{i}(\lambda)$ is in general not an analytic subspace of $\mathcal{V}$; a fortiori its projection $S^{i}(\lambda) \subset S$ is a priori not a complex analytic subvariety of $S$.

Remark 2.4 For $i=0$ and $\lambda \in \mathbb{V}_{\mathbb{Q}}$ the locus $\mathbb{V}^{0}(\lambda)$ is also called the locus of Hodge classes for $\lambda$, usually denoted $\operatorname{Hdg}(\lambda)$; and $S^{0}(\lambda)$ is the Hodge locus of $\lambda$ considered by Weil, namely the locus $\operatorname{HL}(S, \lambda)$ of points of $S$ where some determination of the flat transport of $\lambda$ becomes a Hodge class.

Definition 2.5 Let $\lambda \in \mathcal{V}$.

(a) A component of $\mathbb{V}^{i}(\lambda)$ is an irreducible component of the complex analytic subvariety $\mathbb{V}^{i}(\lambda)$ of the étalé space of the complex local system $\mathbb{V}$.

(b) A component of $S^{i}(\lambda)$ is the image under $p: \mathcal{V} \rightarrow S$ of a component of $\mathbb{V}^{i}(\lambda)$

(c) For $\lambda \in V-\{0\}, i \in \mathbb{Z}$ and $d \in \mathbb{N}$ let $\mathbb{V}^{i}(\lambda)_{\geq d} \subset F^{i} \mathcal{V}$, respectively $S^{i}(\lambda)_{\geq d} \subset S$, be the union of components of $\mathbb{V}^{i}(\lambda)$, resp. $S^{i}(\lambda)$, of dimension at least $d$.

Remark 2.6 Notice that for $\lambda \in \mathcal{V}$ and $z \in \mathbb{C}^{*}, \mathbb{V}^{i}(z \lambda)=z \mathbb{V}^{i}(\lambda)$ and $S^{i}(z \lambda)=$ $S^{i}(\lambda)$ for any $z \in \mathbb{C}^{*}$. Hence, for $\lambda \in \mathcal{V}$ not in the zero section, $S^{i}(\lambda)$ depends only on $[\lambda] \in \mathbb{P} V$.

For $\lambda=\pi\left(\tilde{s}, \lambda_{0}\right) \in \mathcal{V}$ it follows from the theorem of the fixed part (see [23, Cor. 7.23]) that $S^{i}(\lambda) \neq S$ if and only if the $\mathbf{H}_{S}$-orbit of $\lambda_{0}$ in $V$ is not reduced to a point, equivalently if and only if the orbit of $\lambda_{0}$ under $\rho\left(\pi_{1}\left(S, s_{0}\right)\right) \subset$ $\mathbf{G L}(V)$ is infinite. We denote by $\mathbb{V}_{\mathbb{Q}}^{\text {nt }}$ the direct factor of the local system $\mathbb{V}_{\mathbb{Q}}$ corresponding to the sum of non-trivial irreducible $\mathbf{H}_{S}$-factors of $V_{\mathbb{Q}}$ (it is naturally a sub- $\mathbb{Q}$ VHS of $\mathbb{V}_{\mathbb{Q}}$ ). By abuse of notation we write $\mathbb{V}_{\mathbb{Q}}^{\text {nt }}-\{0\}$ for $\mathbb{V}_{\mathbb{Q}}^{\text {nt }}$ with the zero-section removed.

Definition 2.7 We define the locus of non-trivial $F^{i}$-classes

$$
\mathbb{V}_{\geq d}^{i}:=\bigcup_{\lambda \in \mathbb{V}_{\mathbb{C}}^{\mathrm{nt}}-\{0\}} \mathbb{V}^{i}(\lambda)_{\geq d} \subset F^{i} \mathcal{V} \quad \text { and } \quad S^{i}(\mathbb{V})_{\geq d}:=p\left(\mathbb{V}_{\geq d}^{i}\right) \subset S .
$$

Thus the locus of non-trivial (rational) Hodge classes for $\mathbb{V}$ is $\operatorname{Hdg}(\mathbb{V}):=$ $\mathbb{V}_{\mathbb{Q}} \cap \mathbb{V}_{\geq 0}^{0}$ and the Hodge locus $\operatorname{HL}(S, \mathbb{V})$ is $p\left(\mathbb{V}_{\mathbb{Q}} \cap \mathbb{V}_{\geq 0}^{0}\right)$. 


\section{Ingredients and strategy for Theorem 1.5}

Let us now describe the main ingredients and the strategy for the proof of Theorem 1.5. From now on we do not differentiate a complex algebraic variety $X$ from its associated complex analytic space $X^{\text {an }}$, the meaning being clear from the context.

\subsection{On the Zariski-closure of the $F^{i}$-loci}

Given $\lambda \in \mathcal{V}$ and $i \in \mathbb{Z}$ let $\mathbb{V}^{i}(\lambda) \subset \mathcal{V}$ be the locus of $\mathcal{V}$ where the flat transport of $\lambda$ belongs to $F^{i} \mathcal{V}$; and let $S^{i}(\lambda):=p\left(\mathbb{V}^{i}(\lambda)\right) \subset S$ be the locus of points of $S$ where some determination of the flat transport of $\lambda$ at $s$ belongs to $F^{i} \mathcal{V}$, as defined in Sect. 2.

When $i=0$ and $\lambda \in \mathbb{V}_{\mathbb{Q}}$ is rational, $\mathbb{V}^{0}(\lambda)$ is the locus where the flat transport of $\lambda$ is a rational Hodge class. The precise version of Theorem 1.1 is that for $\lambda$ rational, $\mathbb{V}^{0}(\lambda)$ is a closed algebraic subvariety of $\mathcal{V}$, finite over the finite union of special subvarieties $S^{0}(\lambda)$.

To study the Zariski-closure of $\operatorname{HL}\left(S, \mathbb{V}^{\otimes}\right)$ the first idea of this paper consists in studying the geometry of $S^{i}(\lambda)$ for a general, not necessarily rational, $\lambda \in \mathbb{V}_{\mathbb{C}}$. In this generality the subsets $S^{i}(\lambda)$ are usually not even complex analytic subvarieties of $S$, see Sect. 2. However we manage to describe the Zariski-closure of any of their components (see Definition 2.5 for the notion of component of $\left.S^{i}(\lambda)\right)$ :

Theorem 3.1 For any $i \in \mathbb{Z}$ and any $\lambda \in \mathbb{V}_{\mathbb{C}}$, the Zariski-closure of any of the (possibly infinitely many) components of $S^{i}(\lambda)$ is a weakly special subvariety of $S$ for $\mathbb{V}$.

Here the weakly special subvarieties of $S$ for $\mathbb{V}$ are a generalisation, introduced in [15], of the special subvarieties of $S$ for $\mathbb{V}$. See Definition 4.1 for the original definition and Corollary 4.14 for a more geometric description. Theorem 3.1 provides a strong information on components of $S^{i}(\lambda)$ which are of positive period dimension.

Theorem 3.1 is a result in functional transcendence. It follows mainly from the Ax-Lindemann Theorem 4.21 for $\mathbb{Z V H S}$ conjectured in [15, Conj.7.6] as a special case of [15, Conj.7.5], proven by Bakker-Tsimerman [2, Theor. 1.1].

\subsection{A global algebraicity result for the locus of classes of $F^{i}$-type}

The second ingredient in the proof of Theorem 1.5 is a global algebraicity statement for the union of the $F^{i}$-loci of dimension bounded below. Precisely, for any integer $d \geq 0$, let $\mathbb{V}_{\geq d}^{i} \subset F^{i} \mathcal{V}$ be the locus of classes $\lambda \in F^{i} \mathcal{V}$ whose 
orbit under monodromy is infinite and such that $\mathbb{V}^{i}(\lambda)$ is "of dimension at least $d$ at $\lambda$ ", see Definition 2.5. Let $S_{\geq d}^{i}(\mathbb{V})=p\left(\mathbb{V}_{\geq d}^{i}\right)$ be its projection in $S$.

Theorem 3.2 Let $\mathbb{V}$ be a polarized $\mathbb{Z} V H S$ on a smooth quasi-projective variety $S$. For any $i \in \mathbb{Z}$ and any $d \in \mathbb{N}^{*}$, the subset $\mathbb{V}_{\geq d}^{i} \subset F^{i} \mathcal{V}$ is a closed algebraic subvariety of $\mathcal{V}$. Its projection $S^{i}(\mathbb{V})_{\geq d}$ is a closed algebraic subvariety of $S$.

In words: the property of a point $\lambda \in \mathcal{V}$ of having a flat leaf intersecting $F^{i} \mathcal{V}$ in dimension at least $d>0$ is closed in the Zariski-topology. Theorem 3.2 is in fact a special case of a more general result on algebraic flat connections, see Theorem 7.1. It uses in a crucial way the properties of parallel transport.

\subsection{Strategy for the proof of Theorem 1.5}

Let us indicate how Theorem 1.5 follows from Theorem 3.1 and Theorem 3.2.

First, using a finiteness result of Deligne, we are reduced to showing that for $\mathbb{V}$ a polarizable $\mathbb{Z V H S}$ with non-product generic Mumford-Tate group, the Hodge locus of positive period dimension $\operatorname{HL}(S, \mathbb{V})_{\text {pos }}$ is either a finite union of strict special subvarieties of $S$ for $\mathbb{V}$ or is Zariski-dense in $S$.

Let us assume for simplicity that the period map $\Phi_{S}$ for $\mathbb{V}$ is an immersion. In that case the locus of exceptional rational Hodge classes in $\mathcal{V}$ is $\mathbb{V}_{\mathbb{Q}} \cap \mathbb{V}_{\geq 0}^{0}=$ $\mathbb{V}_{\mathbb{Q}} \cap F^{0} \mathcal{V}$; the Hodge locus $\operatorname{HL}(S, \mathbb{V})$ is the projection $p\left(\mathbb{V}_{\mathbb{Q}} \cap \mathbb{V}_{\geq 0}^{0}\right)$; and the Hodge locus of positive period dimension $\operatorname{HL}(S, \mathbb{V})_{\text {pos }}$ is the projection $p\left(\mathbb{V}_{\mathbb{Q}} \cap \mathbb{V}_{\geq 1}^{0}\right) \subset S_{\geq 1}^{0}(\mathbb{V})$. The Zariski-closure $\overline{\operatorname{HL}(S, \mathbb{V})_{\text {pos }}}$ Zar coincides with $p\left(\overline{\mathbb{V}}_{\geq 1}^{0} \cap \mathbb{V}_{\mathbb{Q}} \mathrm{Zar}\right)$.

In Proposition 7.4 we refine Theorem 3.2 to show that there exists a nonempty Zariski open subset $\mathcal{U}$ of ${\overline{\mathbb{V}_{\geq 1}^{0} \cap \mathbb{V}_{\mathbb{Q}}}}^{\text {Zar }} \subset \mathcal{V}$ such that for every point $\lambda \in \mathcal{U}$ there exists a component of $\mathbb{V}^{0}(\lambda)$ of dimension at least 1 contained in $\overline{\mathbb{V}_{\geq 1}^{0} \cap \mathbb{V}_{\mathbb{Q}}}$ Zar . Projecting to $S$, there exists a non-empty Zariski open subset $U$ of $\overline{\mathrm{HL}(S, \mathbb{V})_{\text {pos }}}$ Zar such that for every point $x \in U$ there exists a class $\lambda \in \mathcal{V}$ and a component of $S^{0}(\lambda)$ of dimension at least 1 contained in $\overline{\operatorname{HL}(S, \mathbb{V})_{\text {pos }}}$ Zar and passing through $x$.

By Theorem 3.1 the Zariski-closure of such a component of $S^{0}(\lambda)$ is a weakly special subvariety of $S$ of positive period dimension for $\mathbb{V}$. We thus obtain that there exists a non-empty Zariski open subset $U$ of $\overline{\operatorname{HL}(S, \mathbb{V})_{\text {pos }}}$ Zar such that for every point $x \in U$ there exists a weakly special subvariety $Y_{x}$ of $S$ for $\mathbb{V}$ contained in $\overline{\operatorname{HL}(S, \mathbb{V})_{\text {pos }}}$ Zar and passing through $x$.

Either one of these $Y_{x}$ equals $S$, hence $\overline{\operatorname{HL}(S, \mathbb{V})_{\text {pos }}}$ Zar $=S$. Otherwise the structure of weakly special subvarieties and the assumption that $\mathbf{M T}(S, \mathbb{V})$ 
is non-product imply that each $Y_{X}$ is contained in a strict special subvariety $S_{x}$ of $S$ for $\mathbb{V}$. As such an $S_{x}$ is contained in $\operatorname{HL}(S, \mathbb{V})_{\text {pos }}$ it follows that ${\overline{\operatorname{HL}(S, \mathbb{V})_{\text {pos }}}}^{\mathrm{Zar}}=\operatorname{HL}(S, \mathbb{V})_{\text {pos. }}$. But then $\operatorname{HL}(S, \mathbb{V})_{\text {pos }}$ is a finite union of special subvarieties.

The general case where $\Phi_{S}$ is not a submersion is dealt with similarly using stratifications and the geometry of $S_{d}^{0}(\mathbb{V})$ for all $d \geq 1$.

\subsection{A converse to Theorem 1.1}

Recall that for $\lambda \in \mathbb{V}_{\mathbb{Q}}$ the precise version of Theorem 1.1 states that $\mathbb{V}^{0}(\lambda)$ is a closed algebraic subvariety of $\mathcal{V}$, finite over the finite union of special subvarieties $S^{0}(\lambda)$. As a preliminary to Theorem 3.1, Theorem 3.2 and Theorem 1.5, we also provide for the convenience of the reader the following kind of converse to Theorem 1.1, which might be well-known to experts but which does not seem to have appeared before.

Proposition 3.3 Let $\lambda \in \mathcal{V}$ and $i \in \mathbb{Z}$ be such that $\mathbb{V}^{i}(\lambda)$ is a closed algebraic subvariety of $\mathcal{V}$. Then the projection $S^{i}(\lambda)$ of $\mathbb{V}^{i}(\lambda)$ is a finite union of special subvarieties of $S$. Moreover, $\mathbb{V}^{i}(\lambda)$ is finite over $S^{i}(\lambda)$.

\subsection{Organization of the remaining sections}

Section 4 studies the geometric properties of the weakly special subvarieties of $S$ for $\mathbb{V}$. In particular we prove that they are closed algebraic subvarieties, obtain a key geometric description (Corollary 4.14), prove that they coincide in fact with the bi-algebraic subvarieties of $S$ for the natural bi-algebraic structure on $S$ defined by $\mathbb{V}$ (see Proposition 4.20, a result stated in [15, Prop.7.4] without proof), and state the Ax-Lindemann Theorem 4.21 for them. The following sections provide the proofs of Proposition 3.3, Theorem 3.1, Theorem 3.2 and Theorem 1.5 successively.

\section{Weakly special subvarieties and bi-algebraic geometry for $(S, \mathbb{V})$}

In this section we recall the definition of the weakly special subvarieties of $S$ for $\mathbb{V}$ given in [15], study their geometry and prove their bi-algebraic characterisation (stated in [15] without proof). We recall below the definitions of Hodge theory we need and introduced in [15] (inspired by [21] and [22]), and refer to [15] for more details.

Let $\mathbf{G}$ be the generic Mumford-Tate group of $S$ for $\mathbb{V}$. Any Hodge generic point $s \in S$ defines a morphism of real algebraic groups $h_{s}: \mathbb{C}^{*} \rightarrow \mathbf{G}_{\mathbb{R}}$. All such morphisms belong to the same connected component of a $\mathbf{G}(\mathbb{R})$ conjugacy class $\mathcal{D}$ in $\operatorname{Hom}\left(\mathbb{C}^{*}, \mathbf{G}_{\mathbb{R}}\right)$, which has a natural structure of complex 
analytic space (see [15, Prop.3.1]). The space $\mathcal{D}^{+}$is a so-called MumfordTate domain, a refinement of the classical period domain for $\mathbb{V}$ defined by Griffiths. The pair $\left(\mathbf{G}, \mathcal{D}^{+}\right)$is a connected (pure) Hodge datum in the sense of [15, Section 3.1], called the generic Hodge datum of $\mathbb{V}$. The $\mathbb{Z V H S} \mathbb{V}$ is entirely described by its period map

$$
\Phi_{S}: S \rightarrow \operatorname{Hod}^{0}(S, \mathbb{V}):=\Gamma \backslash \mathcal{D}^{+}
$$

where $\Gamma \subset \mathbf{G}(\mathbb{Z})$ is a finite index subgroup and $\operatorname{Hod}^{0}(S, \mathbb{V}):=\Gamma \backslash \mathcal{D}^{+}$is the associated connected Hodge variety (see [15, Def. 3.18 and below]). We denote by $\tilde{\Phi}_{S}: \tilde{S} \rightarrow \mathcal{D}^{+}$the lift of $\Phi_{S}$.

\subsection{Weakly special subvarieties}

The weakly special subvarieties of $S$ for $\mathbb{V}$ are defined in terms of the weakly special subvarieties of the connected $\operatorname{Hodge}$ variety $\operatorname{Hod}^{0}(S, \mathbb{V})$, which we first recall.

\subsubsection{Weakly special subvarieties of Hodge varieties}

Let $\left(\mathbf{G}, \mathcal{D}^{+}\right)$be a connected Hodge datum and $Y=\Gamma \backslash \mathcal{D}^{+}$an associated connected Hodge variety. Hence $Y$ is an arithmetic quotient in the sense of [3, Section 1] endowed with a natural complex analytic structure (which is not algebraic in general). Recall that a Hodge morphism between connected Hodge varieties is the complex analytic map deduced from a morphism of the corresponding Hodge data (see [15, Lemma 3.9]). The special and weakly special subvarieties of $Y$ are irreducible analytic subvarieties of $Y$ defined as follows (see [15, Def.7.1]):

Definition 4.1 Let $Y$ be a connected Hodge variety.

(1) The image of any Hodge morphism $T \rightarrow Y$ between connected Hodge varieties is called a special subvariety of $Y$.

(2) Consider any Hodge morphism $\varphi: T_{1} \times T_{2} \rightarrow Y$ between connected Hodge varieties and any point $t_{2} \in T_{2}$. Then the image $\varphi\left(T_{1} \times\left\{t_{2}\right\}\right)$ is called a weakly special subvariety of $Y$. It is said to be strict if it is distinct from $Y$.

Remark 4.2 [15, Def.7.1], valid more generally for $Y$ a mixed Hodge variety and generalizing [22, Def.4.1] to this context, gives the following apparently more general definition of a weakly special subvariety. Consider any Hodge morphisms $R \stackrel{\pi}{\leftarrow} T \stackrel{i}{\rightarrow} Y$ between (possibly mixed) connected Hodge varieties and any point $r \in R$. Then any irreducible component of $i\left(\pi^{-1}(r)\right)$ is 
called a weakly special subvariety of $Y$. When $Y$ is pure, i.e. $\mathbf{G}$ is a reductive group, one easily checks that this definition reduces to Definition 4.1(2) above.

Remark 4.3 Considering the connected Hodge variety $T_{2}=\left\{t_{2}\right\}$ associated to the trivial algebraic group, any special subvariety of $Y$ is a weakly special subvariety of $Y$.

Remark 4.4 As noticed in [22, Rem. 4.8] in the case of Shimura varieties, any irreducible component of an intersection of special (resp. weakly special) subvarieties of the Hodge variety $Y$ is a special (resp. a weakly special) subvariety of $Y$. The proof is easy and the details are left to the reader.

\subsubsection{Weakly special subvarieties for $\mathbb{V}$}

As in [15, Prop. 3.20 and Def. 7.1] we define:

Definition 4.5 Let $p: \mathbb{V} \rightarrow S$ be a $\mathbb{Z}$ VHS over a quasi-projective complex manifold $S$ with associated period map $\Phi_{S}: S \rightarrow \operatorname{Hod}^{0}(S, \mathbb{V})$.

Any irreducible complex analytic component of $\Phi_{S}^{-1}(Y)$, where $Y$ is a special (resp. weakly special) subvariety of the connected mixed Hodge variety $\operatorname{Hod}^{0}(S, \mathbb{V})$, is called a special (resp. weekly special) subvariety of $S$ for $\mathbb{V}$. It is said to be strict if it is distinct from $S$.

Notice that an irreducible component of an intersection of special (resp. weakly special) subvarieties of $S$ for $\mathbb{V}$ is not anymore necessarily a special (resp. a weakly special) subvariety of $S$ for $\mathbb{V}$ : it might happen that for $Y \subset$ $\operatorname{Hod}^{0}(S, \mathbb{V})$ a special (resp. weakly special) subvariety the preimage $\Phi_{S}^{-1}(Y)$ decomposes as a union $Z_{1} \cup Z_{2}$ with $Z_{i}, i=1,2$ irreducible; in which case $Z_{1}$ and $Z_{2}$ are special (resp. weakly special) subvarieties in $S$ but an irreducible component of $Z_{1} \cap Z_{2}$ is not. To take this minor inconvenience into account we define more generally:

Definition 4.6 Let $Y \subset \operatorname{Hod}^{0}(S, \mathbb{V})$ be a special (resp. weakly special) subvariety. An irreducible component of the intersection of some irreducible components of $\Phi_{S}^{-1}(Y)$ is called a special (resp. weakly special) intersection in $S$ for $\mathbb{V}$.

The following follows immediately from Remark 4.4:

Lemma 4.7 An irreducible component of an intersection of special (resp. weakly special) intersections for $\mathbb{V}$ is a special (resp. weakly special) intersection for $\mathbb{V}$. 


\subsubsection{Algebraicity of weakly special subvarieties of $S$}

The very definition of the Hodge locus $\operatorname{HL}\left(S, \mathbb{V}^{\otimes}\right)$ implies that special subvarieties of $S$ for $\mathbb{V}$ in the sense of Definition 4.5 coincide with the ones defined in Definition 1.2. In particular, in view of Theorem 1.1, any special subvariety of $S$ (hence any special intersection in $S$ ) is a closed irreducible algebraic subvariety of $S$. An alternative proof of Theorem 1.1 using o-minimal geometry was provided in [3, Theor. 1.6]. The approach of [3] gives immediately the following more general algebraicity result, which is implicit in the discussion of [15, Section 7]:

Proposition 4.8 Any weakly special subvariety $Z$ for $\mathbb{V}$ (hence also any weakly special intersection for $\mathbb{V}$ ) is an algebraic subvariety of $S$.

Proof The proof is strictly analogous to the proof of [3, Theor. 1.6]. By [3, Theor. 1.1(1)] the Hodge variety $\operatorname{Hod}^{0}(S, \mathbb{V})=\Gamma \backslash \mathcal{D}^{+}$is an arithmetic quotient endowed with a natural structure of $\mathbb{R}_{\mathrm{alg}}$-definable manifold. By [3, Theor. 1.3] the period map $\Phi_{S}$ is $\mathbb{R}_{\mathrm{an}, \exp }$-definable with respect to the nat-

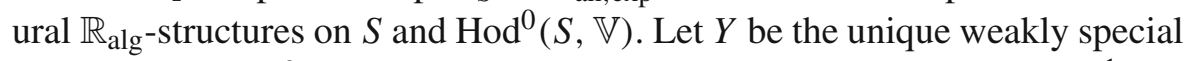
subvariety of $\operatorname{Hod}^{0}(S, \mathbb{V})$ such that $Z$ is an irreducible component of $\Phi_{S}^{-1}(Y)$. By [3, Theor. 1.1(2)] $Y$ is an $\mathbb{R}_{\text {alg }}$-definable subvariety of $\operatorname{Hod}^{0}(S, \mathbb{V})$; hence its preimage $\Phi_{S}^{-1}(Y)$ is an $\mathbb{R}_{\text {an,exp }}$-definable subvariety of $S$. By the definable Chow theorem of Peterzil and Starchenko [20, Theor. 4.4 and Corollary 4.5],

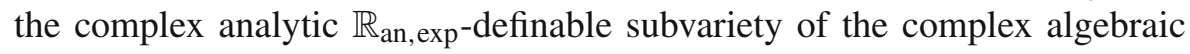
variety $S$ is necessarily an algebraic subvariety of $S$. Hence its irreducible complex analytic component $Z$ too.

\subsubsection{Special and weakly special closure}

One deduces immediately from Lemma 4.7 the following

Corollary 4.9 Any irreducible algebraic subvariety $i: W \hookrightarrow S$ is contained in a smallest weakly special (resp. special) intersection $\langle W\rangle_{\mathrm{ws}}\left(\right.$ resp. $\langle W\rangle_{\mathrm{s}}$ ) of $S$ for $\mathbb{V}$, called the weakly special (resp. special) closure of $W$ in $S$ for $\mathbb{V}$.

Remark 4.10 Obviously $W \subset\langle W\rangle_{\mathrm{ws}} \subset\langle W\rangle_{\mathrm{s}}$.

The geometric description of $\langle W\rangle_{\mathrm{s}}$ is easy. Let $\left(\mathbf{G}_{W}, \mathcal{D}_{W}\right) \subset(\mathbf{G}, \mathcal{D})$ be the generic Hodge datum of the restriction of $\mathbb{V}$ to the smooth locus of $W$. This induces a Hodge morphism of connected Hodge varieties $\varphi: \Gamma_{W} \backslash \mathcal{D}_{W}^{+} \rightarrow$ $\Gamma \backslash \mathcal{D}^{+}$, where $\Gamma_{W}:=\Gamma \cap \mathbf{G}_{W}(\mathbb{Q})$. The restriction of the period map $\Phi_{S}$ to the smooth locus of $W$ factorizes through the special subvariety $\varphi\left(\Gamma_{W} \backslash \mathcal{D}_{W}^{+}\right)$ of $\Gamma \backslash \mathcal{D}^{+}$and we obtain: 
Lemma 4.11 The special closure $\langle W\rangle_{\mathrm{s}}$ is the unique irreducible component of intersections of components of $\Phi_{S}^{-1}\left(\varphi\left(\Gamma_{W} \backslash \mathcal{D}_{W}^{+}\right)\right)$containing $W$.

The description of the weakly special closure $\langle W\rangle_{\mathrm{ws}}$ is a bit more involved but similar to the one obtained by Moonen [19, Section 3] in the case of Shimura varieties. Let $n: W^{\text {nor }} \rightarrow W$ be the normalisation of $W$. Let

$$
\Phi_{W^{\text {nor }}}: W^{\text {nor }} \rightarrow \Gamma_{W} \backslash \mathcal{D}_{W}^{+}
$$

be the period map for $n^{*} \mathbb{V}$. Hence we have a commutative diagram

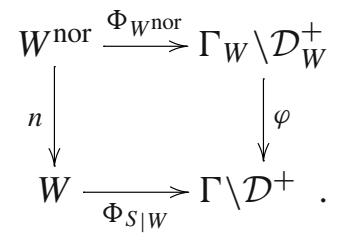

Let $\mathbf{H}_{W}$ be the algebraic monodromy group of $W$ for $\mathbb{V}$. Thus $\mathbf{H}_{W}$ is the identity component of the Zariski-closure of $\left(\Phi_{S} \circ n\right)_{*}\left(\pi_{1}\left(W^{\text {nor }}\right)\right) \subset \Gamma$ in $\mathbf{G L}(V)$. As $W^{\text {nor }}$ is normal the open immersion $j: W^{\text {nor }, 0} \hookrightarrow W^{\text {nor }}$ of the smooth locus $W^{\text {nor, } 0}$ of $W^{\text {nor }}$ defines a surjection $j_{*}: \pi_{1}\left(W^{\text {nor, } 0}\right) \rightarrow \pi_{1}(W)$. In particular $\mathbf{H}_{W}$ is also the algebraic monodromy group of the restriction of $n^{*} \mathbb{V}_{\mathbb{Z}}$ to $W^{\text {nor, } 0}$. It thus follows from [1, Theor.1] that $\mathbf{H}_{W}$ is a normal subgroup of the derived group $\mathbf{G}_{W}^{\mathrm{der}}$. As $\mathbf{G}_{W}$ is reductive there exists a normal subgroup $\mathbf{G}_{W}^{\prime} \subset \mathbf{G}_{W}$ such that $\mathbf{G}_{W}$ is an almost direct product of $\mathbf{H}_{W}$ and $\mathbf{G}_{W}^{\prime}$. In this way we obtain a decomposition of the adjoint Hodge datum $\left(\mathbf{G}_{W}^{\mathrm{ad}}, \mathcal{D}_{W}^{+}\right)$into a product

$$
\left(\mathbf{G}_{W}^{\mathrm{ad}}, \mathcal{D}_{W}^{+}\right)=\left(\mathbf{H}_{W}^{\mathrm{ad}}, \mathcal{D}_{\mathbf{H}_{W}}^{+}\right) \times\left(\mathbf{G}_{W}^{\mathrm{ad}}, \mathcal{D}_{\mathbf{G}_{W}^{\prime}}^{+}\right),
$$

inducing a decomposition of connected Hodge varieties

$$
\Gamma_{W} \backslash \mathcal{D}_{W}^{+}=\Gamma_{\mathbf{H}_{W}} \backslash \mathcal{D}_{\mathbf{H}_{W}}^{+} \times \Gamma_{\mathbf{G}_{W}^{\prime}} \backslash \mathcal{D}_{\mathbf{G}_{W}^{\prime}}^{+} .
$$

Lemma 4.12 The projection of $\Phi_{W^{\text {nor }}}\left(W^{\text {nor }}\right) \subset \Gamma_{W} \backslash \mathcal{D}_{W}^{+}$on $\Gamma_{\mathbf{G}_{W}^{\prime}} \backslash \mathcal{D}_{\mathbf{G}_{W}^{\prime}}^{+}$is a single point $\left\{t^{\prime}\right\}$.

Proof When $\Gamma \backslash \mathcal{D}^{+}$is a connected Shimura variety this is proven in [19, Prop. 3.7]. Moonen's argument does not extend to our more general situation: he uses that $\mathcal{D}^{+}$is a bounded domain in some $\mathbb{C}^{N}$ in the Shimura case, which is not true for a general flag domain $\mathcal{D}^{+}$. Instead we argue as follows. Choose any faithful linear representation $\rho: \mathbf{G}_{W}^{\prime \text { ad }} \rightarrow \mathbf{G L}(H)$ and a $\mathbb{Z}$-structure $H_{\mathbb{Z}}$ 
on the $\mathbb{Q}$-vector space $H$ such that $\rho\left(\Gamma_{\mathbf{G}_{W}^{\prime}}\right) \subset \mathbf{G L}\left(H_{\mathbb{Z}}\right)$. The $\mathbb{Z}$-local system on $W^{\text {nor }}$ with monodromy representation

$$
\lambda: \pi_{1}\left(W^{\mathrm{nor}}\right) \stackrel{\left(\Phi_{W^{\mathrm{nor}}}\right) *}{\longrightarrow} \Gamma_{W} \stackrel{p_{2 *}}{\longrightarrow} \Gamma_{\mathbf{G}_{W}^{\prime}} \stackrel{\rho}{\longrightarrow} \mathbf{G L}\left(H_{\mathbb{Z}}\right)
$$

is a $\mathbb{Z}$ VHS with period map

$$
W^{\text {nor }} \stackrel{\Phi_{W^{\text {nor }, 0}}^{\longrightarrow}}{\longrightarrow} \Gamma_{W} \backslash \mathcal{D}_{W}^{+} \stackrel{p_{2}}{\longrightarrow} \Gamma_{\mathbf{G}^{\prime a d}} \backslash \mathcal{D}_{\mathbf{G}^{\prime}}^{+}
$$

By the very definition of the algebraic monodromy group $\mathbf{H}_{W}$ the group $\lambda\left(\pi_{1}\left(W^{\text {nor }}\right)\right) \subset \mathbf{G L}\left(H_{\mathbb{Z}}\right)$ is finite. Applying the theorem of the fixed part (see [23, Cor. 7.23]) to the corresponding étale cover of $W^{\text {nor }}$ we deduce that the period map $p_{2} \circ \Phi_{W^{\text {nor }}}$ is constant.

Lemma 4.12 implies that $W$ is contained in $\Phi_{S}^{-1}\left(\varphi\left(\left(\Gamma_{\mathbf{H}_{W}} \backslash \mathcal{D}_{\mathbf{H}_{W}}^{+}\right) \times\left\{t^{\prime}\right\}\right)\right)$. Conversely, as any irreducible component of an intersection of weakly special subvarieties of $\Gamma_{W} \backslash \mathcal{D}_{W}^{+}$is still weakly special, one easily checks that any weakly special subvariety $Y:=\psi\left(T_{1} \times\left\{t_{2}\right\}\right) \subset \Gamma_{W} \backslash \mathcal{D}_{W}^{+}$containing $\Phi_{W^{\text {nor }, 0}}\left(W^{\text {nor, }, 0}\right)$ has to contain $\left(\Gamma_{\mathbf{H}_{W}} \backslash \mathcal{D}_{\mathbf{H}_{W}}^{+}\right) \times\left\{t^{\prime}\right\}$. Thus:

Proposition 4.13 The weakly special closure $\langle W\rangle_{\text {ws }}$ of $W$ is the unique irreducible component of the intersection of components of $\Phi_{S}^{-1}\left(\varphi\left(\left(\Gamma_{\mathbf{H}_{W}} \backslash \mathcal{D}_{\mathbf{H}_{W}}^{+}\right) \times\right.\right.$ $\left.\left.\left\{t^{\prime}\right\}\right)\right)$ containing $W$.

It then follows immediately:

Corollary 4.14 The weakly special subvarieties of $S$ for $\mathbb{V}$ (see Definition 4.5) are precisely the closed irreducible algebraic subvarieties $Y \subset S$ maximal among the closed irreducible algebraic subvarieties $Z$ of $S$ whose algebraic monodromy group $\mathbf{H}_{Z}$ with respect to $\mathbb{V}$ equals $\mathbf{H}_{Y}$.

Remark 4.15 The reader will notice that the characterisation of the weakly special subvarieties given above is strictly analogous to the characterisation Definition 1.2 of the special subvarieties, replacing the generic Mumford-Tate group by the algebraic monodromy group.

\subsection{Bi-algebraic geometry for $(S, \mathbb{V})$}

Let us start by recalling the general functional transcendence context of "bialgebraic geometry" (see [17], [15, Section 7]): 
Definition 4.16 A bi-algebraic structure on a connected complex algebraic variety $S$ is a pair

$$
\left(D: \tilde{S} \rightarrow X, \quad \rho: \pi_{1}(S) \rightarrow \operatorname{Aut}(X)\right)
$$

where $\pi: \tilde{S} \rightarrow S$ denotes the universal cover of $S, X$ is a complex algebraic variety, $\operatorname{Aut}(X)$ its group of algebraic automorphisms, $\rho: \pi_{1}(S) \rightarrow \operatorname{Aut}(X)$ is a group morphism (called the holonomy representation) and $D$ is a $\rho$ equivariant holomorphic map (called the developing map).

The datum of a bi-algebraic structure on $S$ tries to emulate an algebraic structure on the universal cover $\tilde{S}$ of $S$ :

Definition 4.17 Let $S$ be a connected complex algebraic variety endowed with a bi-algebraic structure $(D, \rho)$.

(i) An irreducible analytic subvariety $Z \subset \tilde{S}$ is said to be a closed irreducible algebraic subvariety of $\tilde{S}$ if $Z$ is an analytic irreducible component of $D^{-1}\left(\overline{D(Z)}^{\mathrm{Zar}}\right.$ ) (where $\overline{D(Z)}^{\mathrm{Zar}}$ denotes the Zariski-closure of $D(Z)$ in $X)$.

(ii) A closed irreducible algebraic subvariety $Z \subset \tilde{S}$, resp. $W \subset S$, is said to be bi-algebraic if $\pi(Z)$ is a closed algebraic subvariety of $S$, resp. any (equivalently one) analytic irreducible component of $\pi^{-1}(W)$ is a closed irreducible algebraic subvariety of $\tilde{S}$.

As in Sect. 4.1.2 an irreducible component of an intersection of closed algebraic subvarieties of $\tilde{S}$ is not necessarily algebraic in the sense above, as the map $D$ is not assumed to be injective.

Definition 4.18 An algebraic intersection in $\tilde{S}$ is an irreducible analytic component of an intersection of closed algebraic subvarieties of $\tilde{S}$.

An algebraic intersection $Z \subset \tilde{S}$, resp. a closed irreducible algebraic subvariety $W \subset S$, is called a bi-algebraic intersection if $\pi(Z)$ is a closed algebraic subvariety of $S$, resp. any (equivalently one) analytic irreducible component of $\pi^{-1}(W)$ is an algebraic intersection in $\tilde{S}$.

Let $\mathbb{V}$ be a polarized $\mathbb{Z}$ VHS on $S$. It canonically defines a bi-algebraic structure on $S$ as follows. Let

$$
\hat{\Phi}_{S}: \tilde{S} \rightarrow \hat{\mathcal{D}}
$$

be the composite $j \circ \tilde{\Phi}_{S}$ where $j: \mathcal{D} \hookrightarrow \hat{\mathcal{D}}$ denotes the open embedding of the Mumford-Tate domain $\mathcal{D}$ in its compact dual $\hat{\mathcal{D}}$, which is an algebraic flag variety for $\mathbf{G}(\mathbb{C})$ (see [15, Section 3.1]). 
Definition 4.19 Let $p: \mathbb{V} \rightarrow S$ be a polarized $\mathbb{Z V H S}$ on a quasi-projective complex manifold $S$. The bi-algebraic structure on $S$ defined by $\mathbb{V}$ is the pair $\left(\hat{\Phi}_{S}: \tilde{S} \rightarrow \hat{\mathcal{D}}, \rho_{S}:=\left(\Phi_{S}\right)_{*}: \pi_{1}(S) \rightarrow \Gamma \subset \mathbf{G}(\mathbb{C})\right)$.

The following proposition, stated in [15, Prop. 7.4] without proof, characterizes the weekly special subvarieties of $S$ for $\mathbb{V}$ in bi-algebraic terms. It was proven by Ullmo-Yafaev [24] in the case where $S$ is a Shimura variety, and in some special cases by Friedman and Laza [13].

Proposition 4.20 Let $(S, \mathbb{V})$ be a $\mathbb{Z} V H S$. The weakly special subvarieties (resp. the weakly special intersections) of $S$ for $\mathbb{V}$ are the bi-algebraic subvarieties (resp. the bi-algebraic intersections) of $S$ for the bi-algebraic structure on $S$ defined by $\mathbb{V}$.

Proof The proof is similar to the proof of [24, Theor.4.1], we provide it for completeness.

Notice that the statement for the weakly special intersections follows immediately from the statement for the weakly special subvarieties. Hence we are reduced to prove that the weakly special subvarieties of $S$ coincide with the bi-algebraic subvarieties of $S$.

That a weakly special subvariety of $S$ is bi-algebraic follows from the fact that a Hodge morphism of Hodge varieties $\varphi: T \rightarrow Y$ is defined at the level of the universal cover by a closed analytic embedding $\mathcal{D}_{T}^{+} \hookrightarrow \mathcal{D}_{Y}^{+}$restriction of a closed algebraic immersion $\hat{\mathcal{D}}_{T} \hookrightarrow \hat{\mathcal{D}}_{Y}$.

Conversely let $W$ be a bi-algebraic subvariety of $S$. With the notations of Proposition 4.13 the period map $\Phi_{S \mid W}: W \rightarrow \operatorname{Hod}^{0}(S, \mathbb{V})$ factorises trough the weakly special subvariety $\varphi\left(\left(\Gamma_{\mathbf{H}_{W}} \backslash \mathcal{D}_{\mathbf{H}_{W}}^{+}\right) \times\left\{t^{\prime}\right\}\right)$ of $\operatorname{Hod}^{0}(S, \mathbb{V})$. Let $Z$ be an irreducible component of the preimage of $W$ in $\tilde{S}$ and consider the lifting $\tilde{\Phi}_{\mid Z}: Z \rightarrow \mathcal{D}_{\mathbf{H}_{W}}^{+}$of $\Phi_{S \mid W}$ to $Z$. As $W$ is bi-algebraic the Zariski-closure of $\tilde{\Phi}_{\mid Z}(Z)$ in $\hat{\mathcal{D}}_{\mathbf{H}_{W}}$ has to be stable under the monodromy group $\mathbf{H}_{W}(\mathbb{C})$, hence equal to $\hat{\mathcal{D}}_{\mathbf{H}_{W}}$. Thus $Z=\left(\tilde{\Phi}_{\mid Z}\right)^{-1}\left(\mathcal{D}_{\mathbf{H}_{W}}^{+}\right)$and $W$ is weakly special.

We will need the following result, proven for Shimura varieties in [16], conjectured in general in [15, Conj.7.6] as a special case of [15, Conj.7.5], and proven by Bakker-Tsimerman [2, Theor. 1.1]:

Theorem 4.21 (Ax-Lindemann for $\mathbb{Z V M H S ) ~ L e t ~}(S, \mathbb{V})$ be a $\mathbb{Z} V M H S$. Let $Y \subset \tilde{S}$ be a closed algebraic subvariety for the bi-algebraic structure defined by $\mathbb{V}$. Then $\overline{\pi(Y)}$ Zar is a bi-algebraic subvariety of $S$, i.e. a weakly special subvariety of $S$ for $\mathbb{V}$. 


\section{A converse to Theorem 1.1: proof of Proposition 3.3}

Let $f: S^{\prime} \rightarrow S$ be a finite étale cover and let $\mathbb{V}^{\prime}:=f^{*} \mathbb{V}$. By abuse of notation let $f$ still denote the natural map $\mathcal{V}^{\prime} \rightarrow \mathcal{V}$. The reader will immediately check the following (where, with the notations of Sect. 1, we naturally identify $V^{\prime}$ with $V$ ):

Lemma 5.1 (a)

$$
\forall \lambda \in V, \quad \forall i \in \mathbb{Z}, \quad \mathbb{V}^{\prime i}(\lambda)=f^{-1} \mathbb{V}^{i}(\lambda) \text { and } f\left(\mathbb{V}^{\prime i}(\lambda)\right)=\mathbb{V}^{i}(\lambda)
$$

(b) the $f$-image of a special subvariety of $S^{\prime}$ for $\mathbb{V}^{\prime}$ is a special subvariety of $S$ for $\mathbb{V}$; conversely the $f$-preimage of a special subvariety of $S$ for $\mathbb{V}$ is a finite union of special subvarieties of $S^{\prime}$ for $\mathbb{V}^{\prime}$.

Hence proving Proposition 3.3 for $\mathbb{V}$ is equivalent to proving it for $\mathbb{V}^{\prime}$. As any finitely generated linear group admits a torsion-free finite index subgroup (Selberg's lemma) we can thus assume without loss of generality by replacing $S$ by a finite étale cover if necessary that the monodromy $\rho\left(\pi_{1}\left(S, s_{0}\right)\right) \subset$ $\mathbf{G L}\left(V_{\mathbb{Z}}\right)$ is torsion-free.

Let $\lambda \in V-\{0\}$ be such that $\mathbb{V}^{i}(\lambda)$ is an algebraic subvariety of $\mathcal{V}$. Hence $\mathbb{V}^{i}([\lambda]) \subset \mathbb{P} \mathcal{V}$ is also algebraic. As the projection $p: \mathbb{P} \mathcal{V} \rightarrow S$ is a proper morphism, it follows that the set $S^{i}(\lambda):=p\left(\mathbb{V}^{i}([\lambda])\right.$ is an algebraic subvariety of $S$.

Let $n: S^{\prime} \rightarrow S^{i}(\lambda)$ be the smooth locus of the normalisation of one irreducible component of $S^{i}(\lambda)$. Hence $S^{\prime}$ is connected. Let $\pi^{\prime}: \tilde{S}^{\prime} \rightarrow S^{\prime}$ be its universal cover and let $\rho^{\prime}: \pi_{1}\left(S^{\prime}, s_{0}^{\prime}\right) \rightarrow \mathbf{G L}(V)$ be the monodromy of the local system $\mathbb{V}^{\prime}:=i^{-1} \mathbb{V}$ on $S^{\prime}$. Let $\widetilde{\mathbb{V}^{\prime i}(\lambda)}:=\pi^{\prime-1}\left(\mathbb{V}^{\prime i}(\lambda)\right) \subset \widetilde{\mathbb{V}^{\prime}(\lambda)}:=$ $\pi^{\prime-1}\left(\mathbb{V}^{\prime}(\lambda)\right) \simeq \tilde{S}^{\prime} \times\{\lambda\} \subset \widetilde{\mathcal{V}}^{\prime} \simeq \tilde{S}^{\prime} \times V$.

As $\widehat{\mathbb{V}^{\prime}}(\lambda) \subset \tilde{S}^{\prime} \times\{\lambda\}$ and $p:{\mathbb{V ^ { \prime }}}^{i}(\lambda) \rightarrow \tilde{S}^{\prime}$ is surjective, it follows that $\widetilde{\mathbb{V}^{\prime i}(\lambda)}=\tilde{S}^{\prime} \times\{\lambda\}$, hence $\mathbb{V}^{\prime i}(\lambda)=\mathbb{V}^{\prime}(\lambda)$.

In particular $\mathbb{V}^{\prime i}(\lambda) \cap V=\mathbb{V}^{\prime}(\lambda) \cap V=\rho\left(\pi_{1}\left(S^{\prime}, s_{0}^{\prime}\right)\right) \cdot \lambda \subset V$, where $V$ is identified with $\mathcal{V}_{s_{0}^{\prime}}^{\prime}$. As $\mathbb{V}^{\prime i}(\lambda) \subset \mathcal{V}^{\prime}$ is an algebraic subvariety, its fiber $\mathbb{V}^{\prime i}(\lambda) \cap$ $V$ is an algebraic subvariety of $V$. On the other hand the set $\rho\left(\pi_{1}\left(S^{\prime}, s_{0}^{\prime}\right)\right) \cdot \lambda$ is countable. Thus $\mathbb{V}^{\prime i}(\lambda) \cap V$ is a finite set of points, in particular $p: \mathbb{V}^{\prime i}(\lambda) \rightarrow S^{\prime}$ is finite étale.

It follows that the smallest $\mathbb{Q}$-sub-local system $\mathbb{W}_{\mathbb{Q}}^{\prime} \subset \mathbb{V}_{\mathbb{Q}}^{\prime}$ whose complexification $\mathbb{W}^{\prime} \subset \mathbb{V}^{\prime}$ contains $\mathbb{V}^{\prime i}(\lambda)$ has finite monodromy. As the monodromy $\rho\left(\pi_{1}\left(S^{\prime}\right)\right)$ is a subgroup of $\rho\left(\pi_{1}(S)\right.$ which is assumed to be torsion-free, it follows that the local system $\mathbb{W}_{\mathbb{Q}}^{\prime}$ is trivial. By the theorem of the fixed part (see [23, Cor. 7.23]) $\mathbb{W}_{\mathbb{Q}}^{\prime}$ is a constant sub- $\mathbb{Q} V H S$ of $\mathbb{V}^{\prime}$. It follows easily that 
$n\left(S^{\prime}\right)$ is the smooth locus of an irreducible component of the Hodge locus in $S$ defined by the fiber $W_{\mathbb{Q}} \subset V_{\mathbb{Q}}$ of $\mathbb{W}^{\prime}$.

This finishes the proof that $S^{i}(\lambda)$ is a union of special subvarieties of $S$ and that $p: \mathbb{V}^{i}(\lambda) \rightarrow S^{i}(\lambda)$ is finite.

\section{Proof of Theorem 3.1}

Theorem 3.1 follows from Theorem 4.21 and the following

Proposition 6.1 Any component of $S^{i}(\lambda)$ is the image under $\pi$ of an algebraic subvariety of $\tilde{S}$ (for the bi-algebraic structure on $S$ defined by $\mathbb{V}$ ).

Proof of Proposition 6.1 The quadruple $\left(\mathbb{V}_{\mathbb{Z}}, \mathcal{V}, \nabla, F^{\bullet}\right)$ defining the $\mathbb{Z V H S}$ $\mathbb{V}$ is the pullback under $\Phi_{S}$ of a similar quadruple $\left(\mathbb{V}_{\mathbb{Z}, \Gamma \backslash \mathcal{D}^{+}}, \mathcal{V}_{\Gamma \backslash \mathcal{D}^{+}}, \nabla_{\Gamma \backslash \mathcal{D}^{+}}\right.$, $F_{\Gamma \backslash \mathcal{D}^{+}}^{\bullet}$ ) on the connected Hodge variety $\Gamma \backslash \mathcal{D}^{+}$, which however does not satisfy Griffiths transversality. This quadruple itself comes, by restriction to $\mathcal{D}^{+}$and descent to $\Gamma \backslash \mathcal{D}^{+}$, from a $\mathbf{G}(\mathbb{C})$-equivariant quadruple $\left(\mathbb{V}_{\mathbb{Z}, \hat{\mathcal{D}}}, \mathcal{V}_{\hat{\mathcal{D}}}, \nabla_{\hat{\mathcal{D}}}, F_{\hat{\mathcal{D}}}^{\bullet}\right)$ on $\hat{\mathcal{D}}$, see $[10,5.9]$. As $\hat{\mathcal{D}}$ is simply connected the algebraic flat connection $\nabla_{\hat{\mathcal{D}}}$ induces a canonical algebraic trivialization $\mathcal{V}_{\hat{\mathcal{D}}} \simeq \hat{\mathcal{D}} \times V$. Hence we have a commutative diagram

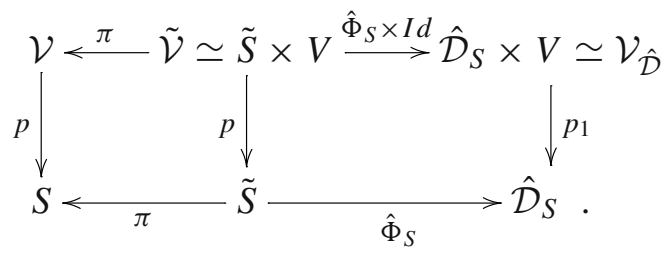

Let $N$ be a component of $S^{i}(\lambda)$. Hence $N=\pi(Y)$, where $Y=p(W)$ for $W \subset \tilde{\mathcal{V}}=\tilde{S} \times V$ an analytic irreducible component of the complex analytic subvariety

$$
\widetilde{\mathbb{V}(\lambda)}:=\left(\hat{\Phi}_{S} \times I d\right)^{-1}\left((\hat{\mathcal{D}} \times\{\lambda\}) \cap F^{i} \mathcal{V}_{\hat{\mathcal{D}}}\right)
$$

of $\tilde{\mathcal{V}}$. Let us define $\hat{\mathcal{D}}(\lambda) \subset \hat{\mathcal{D}}$ as the projection $p_{1}\left((\hat{\mathcal{D}} \times\{\lambda\}) \cap F^{i} \mathcal{V}_{\hat{\mathcal{D}}}\right)$. In particular, corresponding to the diagram (6.1), we have a commutative diagram

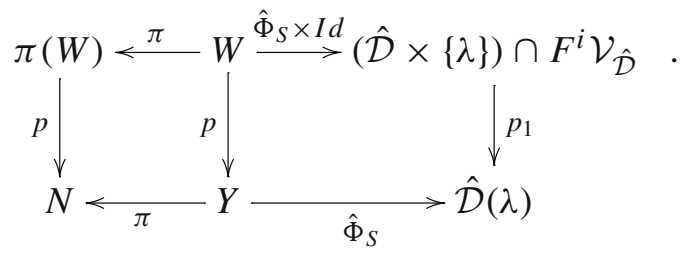


Notice that both $(\hat{\mathcal{D}} \times\{\lambda\})$ and $F^{i} \mathcal{V}_{\hat{\mathcal{D}}}$, hence also their intersection $(\hat{\mathcal{D}} \times$ $\{\lambda\}) \cap F^{i} \mathcal{V}_{\hat{\mathcal{D}}}$, are algebraic subvarieties of $\hat{\mathcal{D}} \times V$. Thus their projection $\hat{\mathcal{D}}(\lambda)$ is an algebraic subvariety of $\hat{\mathcal{D}}$. It follows that the irreducible component $Y$ of $\hat{\Phi}_{S}^{-1}(\hat{\mathcal{D}}(\lambda))$ is an algebraic subvariety of $\tilde{S}$ for the bi-algebraic structure on $S$ for $(S, \mathbb{V})$.

\section{Algebraicity of the $F^{i}$-locus of positive period dimension and relation with the $\mathbb{Q}$-structure}

\subsection{An algebraicity result for flat complex connections}

Theorem 7.1 Let $d$ be a positive integer. Let $p:(\mathcal{V}, \nabla) \rightarrow S$ be an algebraic flat connection on a smooth quasi-projective complex variety $S$ and $\mathbb{V} \subset \mathcal{V}$ the associated complex local system. Let $F \subset \mathcal{V}$ be an algebraic subvariety. For $x \in F$ let $N_{F, x}$ denote the union of irreducible components containing $x$ of the complex analytic subvariety $(\mathbb{V}(x) \cap F)^{\text {red }}$ of the étalé space of $\mathbb{V}$.

The locus $A_{F, \geq d}$ of closed points $x \in F$ such that $N_{F, x}$ has dimension at least $d$ at $x$, is an algebraic subvariety of $\mathcal{V}$.

Proof We write for simplicity $A_{\geq d}:=A_{F, \geq d}$ and $N_{x}:=N_{F, x}$.

Let $T_{h} \mathcal{V} \subset T \mathcal{V}$ denote the horizontal algebraic subbundle of the tangent bundle $T \mathcal{V}$ defined by the flat connection $\nabla$. We write $q: \mathbb{P}(T \mathcal{V}) \rightarrow \mathcal{V}$ for the (proper) natural projection. Let $T_{h} F:=T_{h} \mathcal{V} \times_{T \mathcal{V}} T F$. We define inductively reduced algebraic varieties $\left(A_{\geq d, n}\right)_{n \in \mathbb{N}} \subset \mathcal{V}$ by

- $A_{\geq d, 0}:=F$,

- $A_{\geq d, n+1}:=\left\{x \in A_{\geq d, n} \mid \operatorname{dim}\left(\left(T_{h} A_{\geq d, n}\right)_{x}\right) \geq d\right\}$.

Let $A_{\geq d, \infty}:=\bigcap_{n \in \mathbb{N}} A_{\geq d, n}$. As the $A_{\geq d, n}$ are algebraic subvarieties of $\mathcal{V}$, so is $A_{\geq d, \infty}$.

The result then follows from Lemma 7.2 below.

Lemma 7.2 The equality $A_{\geq d}=A_{\geq d, \infty}$ holds.

Proof The inclusion $A_{\geq d} \subset A_{\geq d, \infty}$ is equivalent to the inclusions $A_{\geq d} \subset$ $A_{\geq d, n}$ for all $n \in \mathbb{N}$, which we show by induction on $n$. By definition $A_{\geq d} \subset$ $F=A_{\geq d, 0}$. Assume that $A_{\geq d} \subset A_{\geq d, n}$ for some $n \in \mathbb{N}$. By definition of $A_{\geq d}$, for any $x \in A_{\geq d}$ the variety $A_{\geq d}$ contains an irreducible component $N$ of $N_{F, x}$ through $x$ of dimension at least $d$. Hence

$$
d \leq \operatorname{dim}(N) \leq \operatorname{dim}\left(T_{x} N\right) \leq \operatorname{dim}\left(\left(T_{h} A_{\geq d, n}\right)_{x}\right),
$$

hence $x \in A_{\geq d, n+1}$. This shows $A_{\geq d} \subset A_{\geq d, n+1}$ and finishes the proof that $A_{\geq d} \subset A_{\geq d, \infty}$. 
Conversely let us prove that $A_{\geq d, \infty} \subset A_{\geq d}$. Let $h: \tilde{\mathcal{V}} \rightarrow V$ denote the composition

$$
h: \tilde{\mathcal{V}} \simeq \tilde{S} \times V \stackrel{p_{2}}{\rightarrow} V
$$

(where the first isomorphism is provided by the flat trivialisation). For $x \in \mathcal{V}$ and $\tilde{x} \in \pi^{-1}(x) \subset \tilde{\mathcal{V}} \simeq \tilde{S} \times V$ let $N_{\tilde{x}}$ be the union of the irreducible components passing through $\tilde{x}$ of the complex analytic subvariety $h^{-1}(h(\tilde{x})) \cap$ $\pi^{-1}(F)$ of $\tilde{\mathcal{V}}$. Thus the local biholomorphism $\pi: \tilde{\mathcal{V}} \rightarrow \mathcal{V}$ identifies $N_{\tilde{x}}$ locally at $\tilde{x}$ with $N_{x}$ locally at $x$.

By noetherianity there exists an $n \in \mathbb{N}$ such that $A_{\geq d, n}=A_{\geq d, n+1}=$ $A_{\geq d, \infty}$. Hence for any $x \in A_{\geq d, \infty}$ we have $\operatorname{dim}\left(\left(T_{h} A_{\geq d, \infty}\right)_{x}\right) \geq d$. Let us consider the restriction

$$
h_{\mid \tilde{A}_{\geq d, \infty}}: \tilde{A}_{\geq d, \infty} \rightarrow V
$$

of $h$ to $\tilde{A}_{\geq d, \infty}:=A_{\geq d, \infty} \times{ }_{S} \tilde{S}$. Let $U_{\geq d, \infty} \subset A_{\geq d, \infty}$ be the Zariski-dense open subset of smooth points $x$ of $A_{\geq d, \infty}$ such that the complex analytic map $h_{\mid \tilde{A}_{\geq d, \infty}}$ is smooth and locally submersive onto its image at any $\tilde{x} \in\{x\} \times{ }_{S} \tilde{S}$. Hence, for $x \in U_{\geq d, \infty}$,

$$
\operatorname{dim}_{\tilde{x}}\left(\tilde{A}_{\geq d, \infty} \times_{V} h(\tilde{x})\right)=\operatorname{dim}\left(\left(T_{h} A_{\geq d, \infty}\right)_{x}\right) \geq d .
$$

Since $U_{\geq d, \infty}$ is Zariski-dense in $A_{\geq d, \infty}$ the inequality $\operatorname{dim}_{\tilde{x}}\left(\tilde{A}_{\geq d, \infty} \times_{V}\right.$ $h(\tilde{x})) \geq d$ holds for any $\tilde{x}$ in the preimage $\tilde{A}_{\geq d, \infty}$ of $A_{\geq d, \infty}$ in $\tilde{\mathcal{V}}$. As $A_{\geq d, \infty} \subset F$, any analytic irreducible component of $\tilde{A}_{\geq d, \infty} \times_{V} h(\tilde{x})$ containing $x$ is contained in $N_{\tilde{x}}$. Thus Equation (7.1) implies that for any $x \in A_{\geq d, \infty}$ we have $\operatorname{dim}_{x}\left(N_{x}\right) \geq d$, i.e. $x \in A_{\geq d}$.

\subsection{Applications to $\mathbb{Q}$-local systems}

The following saturation result will be crucial in the proof of Theorem 1.5:

Proposition 7.3 In the situation of Theorem 7.1 suppose moreover that the local system $\mathbb{V}=\mathbb{V}_{\mathbb{Q}} \otimes_{\mathbb{Q}} \mathbb{C}$ is defined over $\mathbb{Q}$. Let $A_{F, \geq d, \mathbb{Q}}:=A_{F, \geq d} \cap \mathbb{V}_{\mathbb{Q}}$ be the locus of rational classes $x$ whose flat transport meets $F$ at $x$ in dimension $\geq d$ and let $A_{F, \geq d}^{\prime}:={\overline{A_{F, \geq d, \mathbb{Q}}}}^{\mathrm{Zar}}$ be its Zariski-closure in $\mathcal{V}$. There exist a Zariski-open dense subset $U$ of $A_{F, \geq d}^{\prime}$ and, for each $x \in U$, a component $N_{F, x}^{0}$ of $N_{F, x}$ of dimension at least $d$ such that $U \subset \bigcup_{x \in U} N_{F, x}^{0} \subset A_{F, \geq d}^{\prime}$.

Proof As in the proof of Theorem 7.1 we remove from now on the reference to $F$ in our notations. 
First notice that $A_{\geq d, \mathbb{Q}} \subset A_{\geq d}$, hence $A_{\geq d}^{\prime} \subset A_{\geq d}$, as $A_{\geq d}$ is algebraic by Theorem 7.1.

Let $W$ be an irreducible component of $A_{\geq d}^{\prime}$. Since $A_{\geq d+1}^{\prime} \subset A^{\prime} \geq d$, proceeding by decreasing induction on $d$, we may and shall assume that $d$ is the largest integer such that $W \subset A_{\geq d}$. Hence $W \cap A_{\geq d+1}$ is a strict closed algebraic subvariety of $W$. Let $U \subset W$ be the Zariski-open dense subset of all $x \in W-\left(W \cap A_{\geq d+1}\right)$ such that the variety $A_{\geq d}^{\prime}$ is smooth at $x$ and the morphism $h_{\mid \tilde{W}}: \quad \tilde{\tilde{W}} \rightarrow V$ is locally submersive onto its image at any $\tilde{x} \in\{x\} \times{ }_{S} \tilde{S}$. The fibers of the morphism $h_{\mid \tilde{U}}: \tilde{U} \rightarrow V$ are smooth, let us call $D$ their common dimension.

As $U$ is Zariski-open dense in $W$ and $W \cap A_{\geq d, \mathbb{Q}}$ is Zariski-dense in $W$, there exists a point $x_{0} \in U \cap A_{\geq d, \mathbb{Q}}$. The fiber of $h_{\mid \tilde{U}}$ at $\tilde{x}_{0}$ coincides near $\tilde{x}_{0}$ with a component of $N_{\tilde{x}_{0}}$ of dimension d, hence $d=D$.

For any $\tilde{x} \in \tilde{U}$ we have on the other hand

$$
D=\operatorname{dim}_{\tilde{x}}\left(\tilde{W} \times_{H_{s}} \tilde{x}\right)=\operatorname{dim}_{\tilde{x}}\left(N_{\tilde{x}} \cap \tilde{W}\right) \leq \operatorname{dim}_{\tilde{x}}\left(N_{\tilde{x}}\right)=d=D .
$$

Hence for any $x \in U, \operatorname{dim}_{\tilde{x}}\left(N_{\tilde{x}} \cap \tilde{W}\right)=\operatorname{dim}_{\tilde{x}}\left(N_{\tilde{x}}\right)$. Hence there exists a component $N_{x}^{0}$ of $N_{x}$ of dimension $d$ such that $N_{x}^{0} \cap U$ is open and dense in $N_{x}^{0}$. Hence $U$ is dense in $\bigcup_{x \in U} N_{x}^{0}$ (for the usual topology) and $\bigcup_{x \in U} N_{x}^{0} \subset W$. As this holds for any irreducible component $W$ of $A_{\geq d}^{\prime}$, the result follows.

\subsection{Application to $\mathbb{Z}$ VHS: proof of Theorem 3.2 and corollary for Hodge loci}

Suppose now that $\mathbb{V}$ is a $\mathbb{Z}$ VHS and $F=F^{i} \mathcal{V}$. Then $A_{F, \geq d}=\mathbb{V}_{\geq d}^{i}$ and Theorem 7.1 in this case is Theorem 3.2.

Moreover $A_{F, \geq d, \mathbb{Q}}=\mathbb{V}_{\geq d}^{i} \cap \mathbb{V}_{\mathbb{Q}}$ and Proposition 7.3 reads:

Proposition 7.4 Let $S$ be a smooth complex quasi-projective algebraic variety and $\mathbb{V}$ be a polarized $\mathbb{Z} V H S$ over $S$. Let $i \in \mathbb{Z}$ and $d \in \mathbb{N}$. There exist a Zariskiopen dense subset $U$ of $\overline{\mathbb{V}_{\geq d}^{i} \cap \mathbb{V}_{\mathbb{Q}}}$ ar and, for each $\lambda \in U$, a component $\mathbb{V}^{i, 0}(\lambda)$ of $\mathbb{V}^{i}(\lambda)$ of dimension at least d such that $U \subset \bigcup_{\lambda \in U} \mathbb{V}^{i, 0}(\lambda) \subset \mathbb{V}_{\geq d}^{i}$.

Consider now the Zariski-closure $\overline{p\left(\mathbb{V}_{\mathbb{Q}} \cap \mathbb{V}_{\geq d}^{0}\right)}$ Zar . It coincides with the projection $p\left({\overline{\mathbb{V}_{\geq d}^{i} \cap \mathbb{V}_{\mathbb{Q}}}}^{\mathrm{Zar}}\right)$. For $\lambda \in F^{i} \mathcal{V}$ the projection $p\left(\mathbb{V}^{i, 0}(\lambda)\right)$ is a component of dimension at least $d$ of $S^{i}(p(\lambda))$. By Theorem 3.1 the Zariski-closure of any such components is a weakly special subvariety of $S$ of dimension at least $d$. We thus obtain 
Corollary 7.5 Let $S$ be a smooth complex quasi-projective algebraic variety and $\mathbb{V}$ be a polarized $\mathbb{Z} V H S$ over $S$. Let $d \in \mathbb{N}$. Then $\overline{p\left(\mathbb{V}_{\mathbb{Q}} \cap \mathbb{V}_{\geq d}^{0}\right)}$ Zar contains a Zariski-open dense set $U$ with the following property: for each point $x \in U$ there exists a weakly special subvariety $Y_{x} \subset \overline{p\left(\mathbb{V}_{\mathbb{Q}} \cap \mathbb{V}_{\geq d}^{0}\right)}$ Zar of dimension at least d passing through $x$.

\section{Proof of Theorem 1.5}

Following Deligne (see [27, Theor. 4.14] and the comment above it), there exists a bound on the tensors one has to consider for defining $\operatorname{HL}\left(S, \mathbb{V}^{\otimes}\right)$. Thus $\operatorname{HL}\left(S, \mathbb{V}^{\otimes}\right)=\bigcup_{i=1}^{n} \operatorname{HL}\left(S, \mathbb{V}_{i}\right)$ for finitely many irreducible weight zero $\mathbb{Z V H S} \mathbb{V}_{i} \subset \mathbb{V}^{\otimes}$. It follows that $\operatorname{HL}\left(S, \mathbb{V}^{\otimes}\right)_{\text {pos }}=\bigcup_{i=1}^{n} \operatorname{HL}\left(S, \mathbb{V}_{i}\right)_{\text {pos }}$. Hence, replacing $\mathbb{V}$ by $\mathbb{V} \oplus \bigoplus_{i=1}^{n} \mathbb{V}_{i}$ if necessary (this does not change the generic Mumford-Tate group, the period map, or the special subvarieties), we are reduced without loss of generality to showing that for $\mathbb{V}$ a polarizable $\mathbb{Z V H S}$ the Hodge locus of positive period dimension $\operatorname{HL}(S, \mathbb{V})_{\text {pos }}$ is either a finite union of special subvarieties of $S$ for $\mathbb{V}$ or Zariski-dense in $S$.

To make the proof of Theorem 1.5 more transparent we deal first with special cases.

Case 1: the period map $\Phi_{S}$ is an immersion. In that case

$$
\operatorname{HL}(S, \mathbb{V})_{\text {pos }}=p\left(\left(\mathbb{V}_{\mathbb{Q}} \cap(\mathbb{V})_{\geq 1}^{0}\right)\right.
$$

Applying Corollary 7.5 for $d=1$ to $\mathbb{V}$ it follows that $\overline{\operatorname{HL}(S, \mathbb{V})_{\text {pos }}}$ Zar contains a Zariski-open dense subset $U$ with the following property: for each point $x \in U$ there exists a weakly special subvariety $W_{x}$ of positive period dimension for $\mathbb{V}$ passing through $x$ and contained in $\overline{\operatorname{HL}(S, \mathbb{V})_{\text {pos }}}$ Zar

Either there exists $x \in U$ such that $W_{x}=S$, in which case $\overline{\operatorname{HL}(S, \mathbb{V})_{\text {pos }}}{ }^{\text {Zar }}=$ $S$. Or for all $x \in U$ the weakly special subvariety $W_{x}$ of $S$ is strict. In this case the assumption that $\mathbf{M T}(S, \mathbb{V})$ is non-product and the description of weakly special subvarieties given in Sect. 4.1 implies that each $W_{x}$ is contained in a unique strict special subvariety $S_{x}$ of positive period dimension for $\mathbb{V}$. As $S_{x}$ belongs by definition to $\operatorname{HL}(S, \mathbb{V})_{\text {pos }}$, it follows in this case that ${\overline{\mathrm{HL}}(S, \mathbb{V})_{\text {pos }}}^{\mathrm{Zar}}=\mathrm{HL}(S, \mathbb{V})_{\text {pos }}$ is a finite union of strict special subvarieties of $S$, hence the result.

Case 2: the period map $\Phi_{S}$ has constant relative dimension $d$. The proof is the same as in the first case, replacing $\left(\mathbb{V}_{i}\right)_{\geq 1}^{0}$ and "of positive period dimension" by $\left(\mathbb{V}_{i}\right)_{\geq d}^{0}$ and "of period dimension at least $(d+1)$ ".

General case: As the period map $\Phi_{S}$ is definable in the o-minimal structure $\mathbb{R}_{\mathrm{an}, \exp }$ (see [3]), it follows from the trivialization theorem [25, Theor. 
(1.2) p.142] that the locus $S_{d} \subset S$ where the fibers of $\Phi_{S}$ are of complex

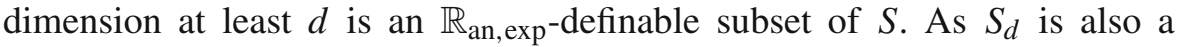
closed complex analytic subset of $S$, if follows from the o-minimal Chow theorem [20, Theor.4.4 and Cor. 4.5] of Peterzil-Starchenko that $S_{d}$ is a closed algebraic subvariety of $S$. Finally we obtain an algebraic filtration $S=S_{d_{0}} \supsetneq S_{d_{1}} \supsetneq \cdots \supsetneq S_{d_{k}} \supsetneq S_{d_{k+1}}=\emptyset$.

Suppose that $\mathrm{HL}(S, \mathbb{V})_{\text {pos }}$ is not algebraic. Let $i \in\{0, \cdots, k\}$ be the smallest integer such that $\left(S_{d_{i}}-S_{d_{i+1}}\right) \cap \mathrm{HL}(S, \mathbb{V})_{\text {pos }}$ is not a closed algebraic subvariety of $S_{d_{i}}-S_{d_{i+1}}$. As $\operatorname{HL}\left(S-S_{d_{i+1}}, \mathbb{V}_{\mid S-S_{d_{i+1}}}^{\otimes}\right)_{\text {pos }}=\operatorname{HL}(S, \mathbb{V})_{\text {pos }} \cap\left(S-S_{d_{i+1}}\right)$, to prove that $\operatorname{HL}(S, \mathbb{V})_{\text {pos }}$ is Zariski-dense in $S$ we can and will assume without loss of generality that $i=k$ (replacing $S$ by $S-S_{d_{i+1}}$ if necessary).

Without loss of generality we can assume that $\operatorname{HL}(S, \mathbb{V})_{\text {pos }}$ is contained in $S_{d_{i}}$ : this is clear if $i=0$, as $S=S_{d_{0}}$ in this case; if $i>0$ there are only finitely many maximal special subvarieties of positive period dimension $Z_{1}, \ldots, Z_{m}$ of $S$ for $\mathbb{V}$ intersecting $S_{d_{i-1}}-S_{d_{i}}$ and we can without loss of generality replace $S$ by $S-\left(Z_{1} \cup \cdots \cup Z_{m}\right)$.

Thus $\operatorname{HL}(S, \mathbb{V})_{\text {pos }}$ coincide with $p\left((\mathbb{V})_{\geq d_{i}+1}^{0} \cap \mathbb{V}_{\mathbb{Q}}\right)$. Applying Corollary 7.5 with $d=d_{i}+1$, it follows that the union $Z$ of irreducible components of $\overline{\mathrm{HL}(S, \mathbb{V})_{\text {pos }}}$ Zar contains a Zariski-open dense set $U$ such that for every point $x \in U$ there exists a weakly special subvariety $W_{x}$ of $S$ for $\mathbb{V}$ of dimension at least $d_{i}+1$ passing through $x$ and contained in $Z$.

If $i>0$ the weakly special subvariety $W_{x} \subset Z \subset S_{d_{i}}$ is strict, and we conclude as above: each $W_{x}$ is contained in a unique strict special subvariety $S_{x}$ of positive period dimension for $\mathbb{V}$, thus $Z=\operatorname{HL}(S, \mathbb{V})_{\text {pos }}$, which contradicts the assumption that $\operatorname{HL}(S, \mathbb{V})_{\text {pos }}$ is not an algebraic subvariety of $S$.

Thus $i=0$. Hence we are in Case 2 above and we conclude that $\operatorname{HL}(S, \mathbb{V})_{\text {pos }}$ is Zariski-dense in $S_{d_{0}}=S$. This finishes the proof of Theorem 1.5.

Acknowledgements We thank O. Benoist and J. Chen for their remarks on this work.

Funding Open Access funding enabled and organized by Projekt DEAL.

Open Access This article is licensed under a Creative Commons Attribution 4.0 International License, which permits use, sharing, adaptation, distribution and reproduction in any medium or format, as long as you give appropriate credit to the original author(s) and the source, provide a link to the Creative Commons licence, and indicate if changes were made. The images or other third party material in this article are included in the article's Creative Commons licence, unless indicated otherwise in a credit line to the material. If material is not included in the article's Creative Commons licence and your intended use is not permitted by statutory regulation or exceeds the permitted use, you will need to obtain permission directly from the copyright holder. To view a copy of this licence, visit http://creativecommons.org/licenses/by/4.0/. 


\section{References}

1. André, Y.: Mumford-Tate groups of mixed Hodge structures and the theorem of the fixed part. Compositio Math. 82, 1-24 (1992)

2. Bakker, B., Tsimerman, J.: The Ax-Schanuel conjecture for variations of Hodge structures. Inventiones Math. Invent. Math. 217(1), 77-94 (2019)

3. Bakker, B., Klingler, B., Tsimerman, J.: Tame topology of arithmetic quotients and algebraicity of Hodge loci. J. Am. Math. Soc. 33(4), 917-939 (2020)

4. Cattani, E., Deligne, P., Kaplan, A.: On the locus of Hodge classes. J. Am. Math. Soc. 8, 483-506 (1995)

5. Cattani, E., Kaplan, A.: Algebraicity of Hodge loci for variations of Hodge structure. In: Hodge Theory, Complex Geometry and Representation Theory, pp. 59-83, Contemporary Mathematics, vol. 608. Amer. Math. Soc., Providence (2014)

6. Chai, C.L.: Density of members with extra Hodge cycles in a family of Hodge structures. Asian J. Math. 2(3), 405-418 (1998)

7. Ciliberto, C., Harris, J., Miranda, R.: General components of the Noether-Lefschetz locus and their density in the space of all surfaces. Math. Ann. 282(4), 667-680 (1988)

8. Clozel, L.: On the cohomology of Kottwitz's arithmetic varieties. Duke Math. J. 72(3), 757-795 (1993)

9. Colombo, E., Pirola, G.P.: Some density results for curves with nonsimple Jacobians. Math. Ann. 288(1), 161-178 (1990)

10. Deligne, P.: Travaux de Griffiths, Semin. Bourbaki 1969/70, No. 376, $213-237$ (1971)

11. Deligne, P.: Equations différentielles à points singuliers réguliers. LNM 163 (1970)

12. Edixhoven, B., Yafaev, A.: Subvarieties of Shimura varieties. Ann. Math. 157(2), 621-645 (2003)

13. Friedman, M., Laza, R.: Semialgebraic horizontal subvarieties of Calabi-Yau type. Duke Math. J. 162(12), 2077-2148 (2013)

14. Izadi, E.: Density and completeness of subvarieties of moduli spaces of curves or abelian varieties. Math. Ann. 310(2), 221-233 (1998)

15. Klingler, B.: Hodge theory and atypical intersections: conjectures. arXiv:1711.09387, to appear in the book Motives and complex multiplication, Birkhaüser

16. Klingler, B., Ullmo, E., Yafaev, A.: The hyperbolic Ax-Lindemann-Weierstraß conjecture. Publ. Math. IHES 123(1), 333-360 (2016)

17. Klingler, B., Ullmo, E., Yafaev, A.: Bi-algebraic geometry and the André-Oort conjecture. In: Proceedings of 2015 AMS Summer Institute in Algebraic Geometry, PSPMS 97-2, pp. 319-360. AMS (2018)

18. Milne, J.: Introduction to Shimura varieties. In: Harmonic Analysis, the Trace Formula and Shimura Varieties (James Arthur, Robert Kottwitz, Editors) AMS, 2005, (Lectures at the Summer School held at the Fields Institute, June 2-June 27, 2003). An expanded version is available for free download on author's web-page

19. Moonen, B.: Linearity properties of Shimura varieties. I. J. Algebraic Geom. 7, 539-567 (1998)

20. Peterzil, Y., Starchenko, S.: Complex analytic geometry and analytic-geometric categories. J. Reine Angew. Math. 626, 39-74 (2009)

21. Pink, R.: Compactification of mixed Shimura varieties. Dissertation, Bonner Math. Schriften 209

22. Pink, R.: A combination of the conjectures of Mordell-Lang and André-Oort. In: Geometric Methods in Algebra and Number Theory, Progress in Mathematics, vol. 253, pp. 251-282. Birkhaüser (2005)

23. Schmid, W.: Variation of Hodge structure: the singularities of the period mapping. Invent. Math. 22, 211-319 (1973) 
24. Ullmo, E., Yafaev, A.: A characterization of special subvarieties. Mathematika 57, 263-273 (2011)

25. van den Dries, L.: Tame Topology and o-Minimal Structures. LMS Lecture Note Series, 248. Cambridge University Press, Cambridge (1998)

26. Voisin, C.: Théorie de Hodge et géométrie algébrique complexe, Cours Spécialisés 10. Société Mathématique de France, Paris (2002)

27. Voisin, C.: Hodge loci. In: Handbook of Moduli. Advanced Lectures in Mathematics, 26, vol. III, pp. 507-546. Int. Press, Somerville, MA (2013)

28. Weil, A.: Abelian Varieties and the Hodge Ring, Collected Papers III, pp. 421-429. Springer, Berlin (1979)

29. Zannier, U.: Some problems of unlikely intersections in arithmetic and geometry. With appendixes by David Masser. Annals of Mathematics Studies, vol. 181, PUP (2012)

Publisher's Note Springer Nature remains neutral with regard to jurisdictional claims in published maps and institutional affiliations. 